\title{
Forced and mixed convection experiments in a confined vertical backward facing step at low-Prandtl number
}

\author{
Thomas Schaub $^{1}\left[\right.$ ] Frederik Arbeiter ${ }^{1} \cdot$ Wolfgang Hering $^{1} \cdot$ Robert Stieglitz $^{1}$
}

Received: 14 July 2021 / Revised: 31 October 2021 / Accepted: 29 November 2021 / Published online: 29 December 2021

(c) The Author(s) 2021

\begin{abstract}
In this paper, we present experimental results for a non-isothermal vertical confined backward facing step conducted with a low-Prandtl number fluid. The eutectic alloy gallium-indium-tin is used as the working fluid. We conducted experiments for different Reynolds and Richardson numbers covering both forced and mixed convection regimes. Time-averaged velocity profiles were measured at six streamwise positions along the test section center-plane with so-called permanent magnet probes. The local Nusselt number was measured in streamwise and spanwise directions along the heating plate mounted right after the step. We further ran RANS simulations of the experiment to study the qualitative influence of assuming a constant specific heat flux thermal boundary condition for the experiment heating plate. The measured velocity profiles show the expected behavior for both studied convection regimes, while the measured streamwise local Nusselt number profiles do not. This is explained by how the heating plate thermal boundary condition is defined. We performed an order of magnitude estimate to estimate the forced- to mixed convection transition onset. The estimate shows good agreement with the experimental data, although further measurements are needed to further validate the estimated transition threshold. The measurement of fluctuating quantities remains an open task to be addressed in future experiments, since the permanent magnet probe measurement equation needs further adjustments.
\end{abstract}

$\begin{array}{ll}\text { Abbreviations } \\ \text { AR } & \text { Aspect ratio } \\ \text { BFS } & \text { Backward facing step } \\ \text { ER } & \text { Expansion ratio } \\ \text { DNS } & \text { Direct numerical simulation } \\ \text { GaInSn } & \text { Gallium-indium-tin } \\ \text { GUM } & \text { Guide to the Expression of Uncertainty in } \\ & \text { Measurement } \\ \text { PEEK } & \text { Polyether ether ketone } \\ \text { PMP } & \text { Permanent magnet probe } \\ \text { RANS } & \text { Reynolds-averaged Navier-Stokes } \\ \text { TC } & \text { Thermocouple }\end{array}$

Thomas Schaub thomas.schaub@kit.edu

1 Institute for Neutron Physics and Reactor Technology (INR), Karlsruhe Institute of Technology (KIT), Hermann-von-Helmholtz-Platz 1, Eggenstein-Leopoldshafen, Germany

\section{Introduction}

The numerical solution of the Reynolds-averaged Navier-Stokes equations (RANS) is the preferred method in industry for the numerical calculation of heat transfer related problems. In order for these models to properly predict heat transfer rates and wall temperatures, turbulent heat fluxes $u_{i}^{\prime} T^{\prime}$ must be accurately calculated. The vast majority of available validation data for $u_{i}^{\prime} T^{\prime}$-models cover Prandtl numbers $(P r)$ of the order of unity, while experimental data for very low $\operatorname{Pr}(\operatorname{Pr} \ll 1)$ fluids are rare. The main reason is due to intrinsic complications and challenges related to the design and operation of liquid metal facilities and the respective instrumentation, particularly for the local and simultaneous measurement of the temperature and velocity of the flow. Within this context, the preferred turbulent heat flux model validation methodology for $\operatorname{Pr} \ll 1$ flows is to validate the models against DNS data.

The available experimental data for the validation of $P r \ll 1$ turbulent heat flux models correspond to canonical flow geometries such as vertical pipe flow (Weissenfluh (1985)), a confined vertical jet in a co-flow (Knebel et al. 1998) and a horizontal mixing layer flow (Kapulla (2000)). The next step is to validate available turbulence 
and turbulent heat flux models in more complex flow configurations. Although experimental results for more complex geometries such as rod-bundle flow are available (Weissenfluh (1988), Pacio et al. (2019)), due to their complexity and difficult instrumentation accessibility, they can only provide information for local temperature distributions and pressure losses. A non-isothermal backward facing step (BFS) flow is a complex flow configuration that also enables the local measurement of both temperatures and velocity profiles with so-called permanent magnet probes (Schaub et al. (2021)). Alternatively, one may think of a BFS as the superposition of some canonical flows: wall bounded flow, buoyancy aided flow, recirculating flow, mixing layer flow, adverse pressure-gradient flow and boundary layer growth under non-equilibrium conditions. All of these can be found in relevant industrial applications (concentrated solar power plants, modern production of radioisotopes for medical applications, hydrogen production by methane pyrolysis, among others). That is, if a turbulence and turbulent heat flux model can accurately predict the flow and heat transfer phenomena in a non-isothermal BFS, one may use those models for industrial flow applications that may be thought of as a the superposition of the respective canonical flows.

As to our best knowledge, the present experiment is the first very low Prandtl $(P r \ll 1)$ BFS experiment available in the open literature. The only BFS experiment for moderately low Prandtl $(\mathrm{Pr}<1)$ BFS available in the open literature is that of Buckingham (2018). Their working fluid was a mixture of helium and xenon to achieve lower $\operatorname{Pr}$ than that of air. A vast literature summary of all isothermal and nonisothermal BFS experiments and direct numerical simulations (DNS) available in the literature (mainly for $\mathrm{Pr} \sim 1$ ) can be found in Chen et al (2018). Nadge and Govardhan (2014) present an analysis of past and own experimental results $(P r \sim 1)$ on the dependence of the BFS recirculation region structure on geometrical and flow parameters.

In the absence of any other BFS experiments for $P r \ll 1$ in the open literature, we present a short literature review on the numerical work done in the past years for $\operatorname{Pr} \ll 1$ BFS. Niemann and Fröhlich (2016a) ran the first DNS for a $P r \ll 1$ BFS flow available in the literature. They simulated a non-confined BFS (on the definition of non-confined, see Sect. 2.2) using sodium as a working fluid $\left(\mathrm{Pr}=O\left(10^{-3}\right)\right.$ for both forced- and mixed convection regimes at a Reynolds number of $R e_{h}=4805$, based on the inlet bulk velocity and the step height. Niemann \& Fröhlich $(2016 a, b)$ DNS dataset triggered many turbulence and turbulent heat flux model validation cases (Schumm et al. (2018), De Santis and Shams (2018) and Da Vià and Manservisi (2019)). Oder et al. (2019) ran the first confined BFS DNS for a $P r \ll 1$ for the forced convection regime. Wang et al. (2020) conducted different DNS for non-confined BFS, also for mixed- and forced convection regimes but for lead-bismuth
$\left(\operatorname{Pr}=O\left(10^{-2}\right)\right)$. Recently, Star et al. (2021) developed a reduced order model for the calculation of a non-confined BFS for liquid sodium.

The present paper shows the results for an experiment that presents results for a $P r \ll 1\left(P r=O\left(10^{-2}\right)\right)$ confined backward facing step for both forced and mixed convection regimes. This experiment is a preceding step to a BFS experiment to be conducted in a large sodium facility (Jäger et al. (2017)) and.

This paper is organized as follows. The experimental facility, the backward facing step test section and its boundary conditions are described and characterized in Sect. 2. Section 3 outlines the developed instrumentation and measurement chain for the simultaneous and local measurement of temperature and velocity. In Sect. 4, the experimental campaign parameter set is discussed together with the limitations of the experiment. The experimental results for the measured velocity profiles and local Nusselt number along the heating plate are presented in Sect. 5. In Sect. 6, a criterion for the transition from forced to mixed convection is derived based on a rough order of magnitude estimate of the equations of fluid motion. The main conclusions are summarized in Sect. 7, and the uncertainty analysis is shown in the appendix 10.

\section{Experimental setup}

\subsection{Facility}

A sketch of the experimental facility is shown in Fig. 1. A detailed description of the facility was treated in Schaub et al. (2021).

The working fluid of the facility is the non-toxic eutectic alloy of gallium-indium-tin ( $G a I n S n)$. Its thermo-physical and some electrical properties are shown in Table 1, where $\rho$ is the density, $\mu$ is the dynamic viscosity, $\nu$ is the kinematic viscosity, $c_{p}$ is the specific heat at constant pressure, $k$ is the thermal conductivity, $\alpha$ is the thermal diffusivity, $\gamma$ is the surface tension, and $\sigma$ is the electric conductivity.

The liquid metal flows-according to Fig. 1-in clockwise direction. The facility consists of mainly four sections: (i) the pumping section, where the liquid metal is pumped by a permanent magnet pump (SAAS GmbH); (ii) the flow conditioning section, where all upstream flow disturbances are suppressed to an acceptable minimum; (iii) the confined backward facing step test section, to be described more in detail in Sect. 2.2; and (iv) the return section, where a water-GaInSn counterflow heat exchanger and the flow meter (ABB Hygenic Master inductive flow meter) are mounted. The flow conditioning section was designed according to well-known wind- and water-tunnel design guidelines. All 


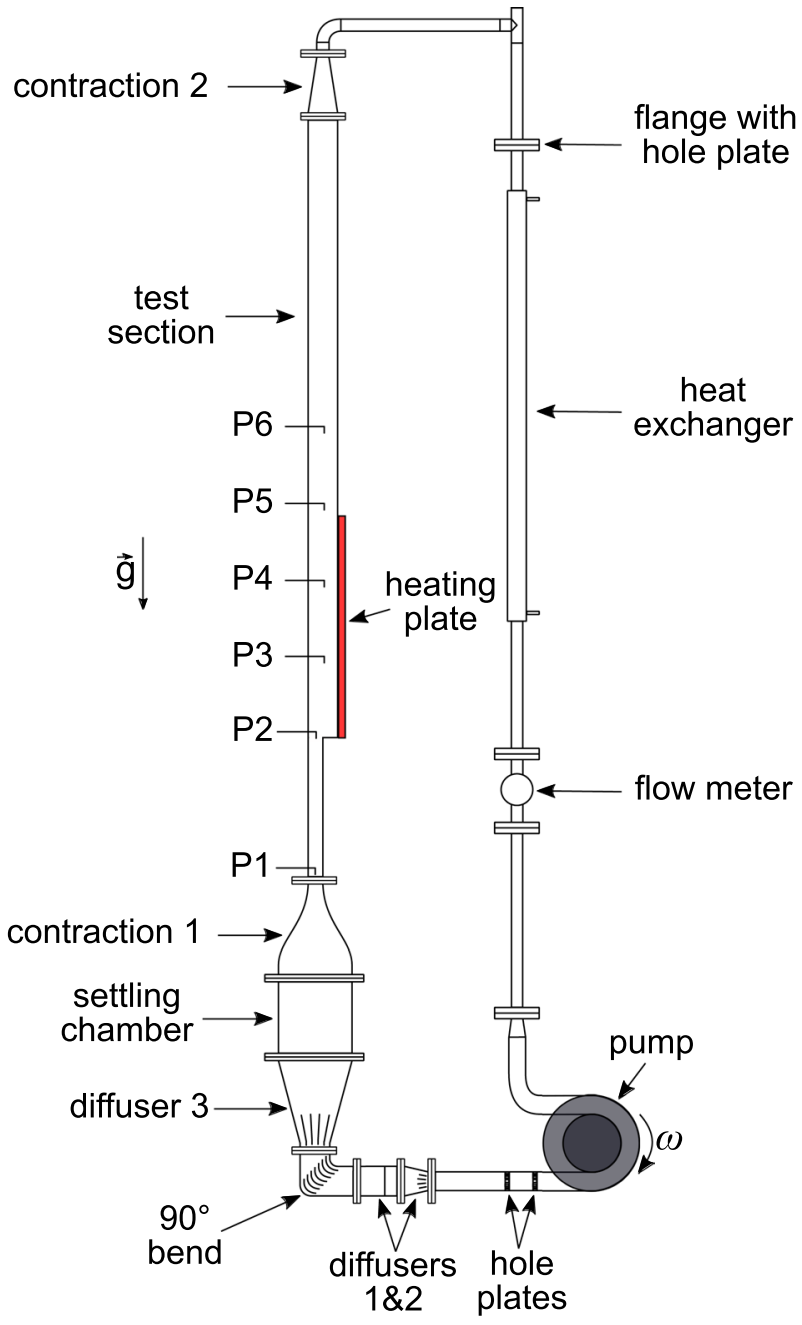

Fig. 1 Sketch (to scale) of the experimental facility

Table 1 Thermophysical properties of the working fluid galliumindium-tin (GaInSn) taken from Plevachuck (2014).. For comparison, the properties for liquid sodium (Sobolev (2011) and water (NIST) are also shown

\begin{tabular}{llll}
\hline & $\begin{array}{l}\text { Gallium-indium- } \\
\text { tin } \mathrm{GaInSn}\end{array}$ & Sodium $\mathrm{Na}$ & Water $\mathrm{H}_{2} \mathrm{O}$ \\
\hline$T_{r e f}\left({ }^{\circ} \mathrm{C}\right)$ & 25 & 200 & 25 \\
$\rho\left(\mathrm{kg} / \mathrm{m}^{3}\right)$ & 6412 & 903 & 997 \\
$\mu(\mathrm{mPa} \cdot \mathrm{s})$ & 2.10 & 0.45 & 0.89 \\
$\nu\left(\mathrm{m}^{2} / \mathrm{s}\right)$ & $0.32 \cdot 10^{-6}$ & $0.50 \cdot 10^{-6}$ & $0.89 \cdot 10^{-6}$ \\
$c_{p}(\mathrm{~J} / \mathrm{kg} \cdot \mathrm{K})$ & 400 & 1343 & 4218 \\
$\lambda(\mathrm{W} / \mathrm{K} \cdot \mathrm{m})$ & 25.06 & 81.94 & 0.68 \\
$\alpha\left(\mathrm{m}^{2} / \mathrm{s}\right)$ & $9.78 \cdot 10^{-6}$ & $67.57 \cdot 10^{-6}$ & $0.16 \cdot 10^{-6}$ \\
$\operatorname{Pr}(-)$ & 0.034 & 0.007 & 5.523 \\
$\gamma(\mathrm{N} / \mathrm{m})$ & 0.59 & 0.19 & 0.07 \\
$\sigma(1 / \Omega \cdot m)$ & $3.25 \cdot 10^{6}$ & $7.70 \cdot 10^{6}$ & $5.49 \cdot 10^{-6}$ \\
\hline
\end{tabular}

the facility components but the pump and the flow meter were manufactured in-house.

\subsection{Test section}

The mounted test section is a vertical confined backward facing step (BFS) with a heating plate right after the step (Fig. 2). The dimensions of the test section scaled by the step height $h=0.04 m$ as well. A BFS is said to be confined depending on its aspect ratio $(A R)$, i.e., the ratio between the channel span and the step height. If $A R>10$, a BFS is said to be non-confined, i.e., one can assume that the flow at the center-plane along the BFS will not be influenced by the induced secondary motions of the second kind at the corners (de Brederode and Bradshaw 1972). Contrary, if $A R<10$, a BFS is said to be confined, i.e., secondary motions of the second kind must be considered in the analysis throughout the entire flow domain. A systematic analysis on the influence of secondary motions as a function of aspect ratio for plane channel flows can be found in Vinuesa et al. (2018). For this experiment, $A R=2$. Another relevant parameter to characterize a BFS is its expansion ratio $(E R)$, i.e., the ratio between the outlet section channel height and the step height, which for this test section is $E R=2$.

The test section was manufactured based on a doublewall-channel concept: an inner channel material made of polyetheretherketone (PEEK) enveloped by an external channel made of stainless steel. Right after the BFS-step, a copper plate with its side walls framed in PEEK was inserted into the test section serving both as the fluid wall in that region and as a heating surface. In the inlet section, a trip wire surrounds all four channel walls. The reason
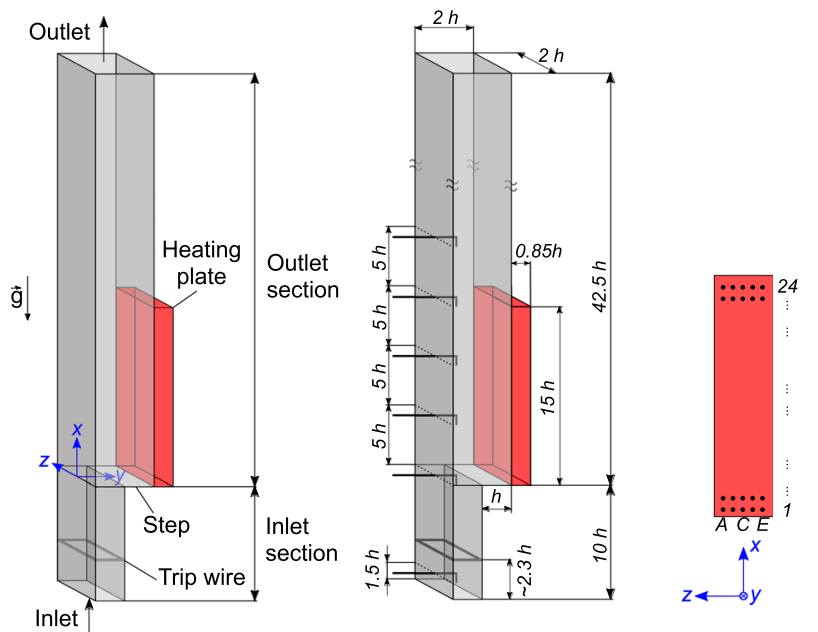

Fig. 2 Test section (not to scale). All dimensions are expressed in dimensionless units taking the step height as a reference, $h=0.04(\mathrm{~m})$. The probes are installed along the test section center-plane. The indicated coordinate system is used from now on for all figures 
behind these design measures is explained more in detail in Sect. 2.3.

Along the test section, six so-called permanent magnet probes (PMP) allow the local and simultaneous measurement of the fluid temperature and velocity at the test section central plane. The local temperature near the interface between the copper heating plate and the liquid metal is measured with 120 type $K$ thermocouples arranged in five columns ( $A$ to $E$ ), each with 24 thermocouples. They are guided from outside through the heating plate up to $0.5 \mathrm{~mm}$ to the heating plate contact surface to the liquid metal (see Fig. 13).

\subsection{Analysis and characterization of the experimental boundary conditions}

\subsubsection{Inlet and outlet}

The BFS flow is sensitive to its boundary conditions (Nadge and Govardhan (2014). The ideal scenario is to place the BFS inlet and outlet as far away as possible from the region of interest, i.e., the recirculating region right after the step. On the one hand, this facilitates the comparison of the experimental results with numerical calculations, since the generation of a fully developed turbulent inlet profile in numerical calculations is straightforward. On the other hand, due to the elliptic nature of pressure fields, any disturbance near the experiment outlet will be felt by the upstream flow. By placing the outlet as far away as possible, these disturbances can be reduced down to a minimum. However, due to the limited available amount of liquid metal in our laboratory, a compromise had to be made regarding the extension of the inlet and the outlet sections. Inlet disturbances are managed by means of well-known flow conditioning techniques (hole plates, screens, honeycombs), while letting any outlet disturbances dissipate by extending the outlet section as far downstream as possible. Furthermore, this also justifies the assumption of a nearly zero-pressure gradient along the outlet section past a certain point after the step. From own numerical simulations, this corresponds to about two times the mean reattachment length. In other words, the complete outlet section extension of the test section does not need to be considered in a numerical simulation. The test section dimensions are shown in Fig. 2, where all dimensions are scaled by the step height, $h=40 \mathrm{~mm}$.

A constant or flat velocity profile is required at the test section inlet to calibrate the permanent magnet probes at the calibration position $P 1$ (Fig. 1). The velocity magnitude is obtained from the continuity equation taking the experiment flow rate measurements and then correlating these values with the induced probe voltages. The turbulence intensity $T u$ at position $P 1$ is estimated as $1 \%<T u<2 \%$ (Schaub et al. (2021)). Since at the test section inlet a very thin and eventually relaminarized boundary layer cannot be discarded, a trip wire is placed after the calibration position to trigger at a single position the boundary layer transition from laminar to turbulent. Furthermore, as it is known, turbulent boundary layers grow faster than laminar ones. This enables a thicker and hence an easier-to-measure boundary layer at position $P 2$ in Fig. 1. The trip wire has a square cross section of $2 m m x 2 \mathrm{~mm}$ and its relative position to the test section inlet is shown in Fig. 2.

The temporal stability of the temperature inlet boundary condition is given by the interaction between the heat sources and heat sinks of the facility, which can only be accurately assessed in practice during the commissioning phase of the facility. In the experiment, the inlet temperature showed a maximum peak-to-peak fluctuation of $\sim 0.5^{\circ} \mathrm{C}$ for the lowest heating plate input power parameters. Unfortunately, these fluctuations are in same order of magnitude as the actual measured temperature gradients in the fluid. One alternative to eliminate the inlet temperature variability is to zero-averaging them by taking longer measurements. This must be done for every single of the 114 measurement points for each parameter set. This is not feasible in practice. Hence, at this stage of the project, we are not able to show the results for the temperature profiles in the fluid. However, as will be shown later in Sect. 4.2.2, the zero-averaging strategy was successfully implemented for the measurement of the heating plate local temperature.

\subsubsection{Walls}

All walls were mechanically wetted with $G a I n S n$ by rubbing it with a sponge to the walls. The wetting-procedure result is illustrated in Fig. 3, where the picture was taken from the outlet into the test section. A comparison between pressure drop measurements (of rather qualitative character-see

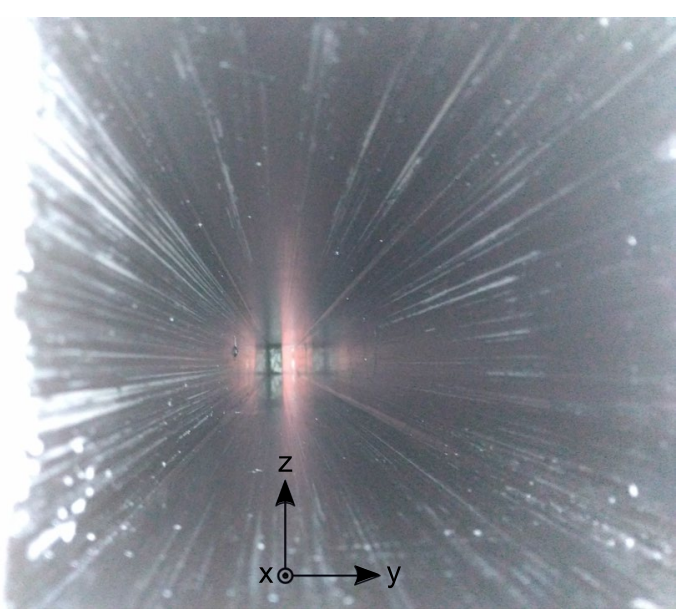

Fig. 3 Picture of the mechanically wetted test section taken from the outlet into the test section 
below) along the test section and complementary numerical simulations plus a comparison with empirical correlations (Idelchik (2008)) show good agreement. The no-slip boundary condition is hence assumed.

The position of the pressure measurement points is not shown in Fig. 2. During the facility commissioning, we noticed liquid metal leaks in the pressure transducers. For this reason, we unmounted them and were not able to measure the pressure drop along the test section during the actual measurement campaign.

The test section walls are considered adiabatic, since the inner walls of the test section channel were manufactured of $\operatorname{PEEK}\left(\lambda_{\text {PEEK }}=0.29 \mathrm{~W} / \mathrm{K} \cdot \mathrm{m}\right)$ and the test section external channel was further insulated with $\sim 40 \mathrm{~mm}$ (Armaflex $\AA$, $\left.\lambda_{\text {Armaflex }}=0.033 \mathrm{~W} / \mathrm{K} \cdot \mathrm{m}\right)$.

\subsubsection{Heating plate}

What in Fig. 2 is referred to as the "heating plate," is actually a heating structure consisting of two copper blocks: the inner and the outer block. In Fig. 2, the total thickness of the heating structure is indicated. The inner block is mounted into the test section and works as the contact surface to the liquid metal. The inner block has the same wall thickness as the PEEK inner- and stainless steel outer-walls together, i.e., it overlaps both walls. To avoid axial thermal losses to the test section stainless steel outer wall, the inner copper block is thermally insulated over its perimeter with a PEEK frame. The sensing thermocouples are integrated into the inner copper block. The outer copper block is the actual heating plate and is pressed onto the inner block. To guarantee a homogeneous thermal contact between both blocks, we decided not to use thermal paste, but to wring the outer block onto the inner block (as done with gauge blocks) and then mechanically press it. Electrical steel housed resistance heaters are integrated within the outer copper block. The external open surface is thermally insulated with a $\sim 100 \mathrm{~mm}$ high temperature insulating wool layer, which at the same time is housed in a $\sim 40 \mathrm{~mm}$ thick Armaflex ${ }^{\circledR}$ box.

The heating plate thermal boundary condition type can be assessed by calculating the heating plate Biot number.

$B i=\frac{\alpha l_{H P}}{\lambda_{H P}}$

where $\alpha$ is the heat transfer coefficient on the heating plate, $\lambda_{H P}$ is the thermal conductivity of the heating plate (in this case copper, with $\lambda_{H P}=401 \mathrm{~W} / \mathrm{K} \cdot \mathrm{m}$ ), and $l_{H P}$ is the characteristic length of the heating plate calculated as $l_{H P}=V_{H P} / A_{H P}$, where $V_{H P}$ is the heating plate volume and $A_{H P}$ is the heating plate contact surface to the liquid metal.

The heat transfer coefficient $\alpha$ along the heating plate is defined as.
$\alpha=\frac{\dot{q}}{T_{w}-T_{b}}$

where $\dot{q}$ is the heating plate specific heat flux, $T_{w}$ the heating plate wall temperature, and $T_{b}$ a case-specific ad hoc bulk temperature, in this case $T_{b}=T_{\text {inlet }}$, where $T_{\text {inlet }}$ is the test section inlet temperature.

The Nusselt number is calculated as.

$N u=\frac{\alpha l_{c}}{\lambda_{\text {ref }}}=\frac{\dot{q} l_{c}}{\lambda_{\text {ref }}\left(T_{w}-T_{b}\right)}$

where $l_{c}$ is the characteristic length (in this case the step height), and $\lambda_{\text {ref }}$ the thermal conductivity of the working fluid at the reference temperature $T_{r e f}=25^{\circ} \mathrm{C}$ (in this case GaInSn).

For all measured cases, the estimated Biot number is in the order $B i=O\left(10^{-1}\right)$. This is an indication that a constant heat flux thermal boundary condition cannot be assumed for the heating plate. A mixed-type thermal boundary condition is more likely to describe the actual thermal boundary condition. This means that - theoretically—not a constant specific heat flux $\dot{q}_{\text {const }}$ but a local one $\dot{q}_{\text {local }}$ should be used to calculate $N u$ in Eq. 3. When calculating $N u$ assuming either way a constant specific heat flux $\dot{q}_{c o n s t}, N u$ can then only be interpreted as a quantity proportional to the reciprocal of the local $\Delta T=T_{w}-T_{b}$. In future experiments, $\dot{q}_{\text {local }}$ will be measured or, alternatively, it will be calculated by solving the inverse Fourier problem from local temperature measurements (Woodfield et al. (2006)).

For the present experimental campaign, the constant specific heat flux $\dot{q}_{\text {const }}$ is used to determine $N u$ and is calculated as $\dot{q}_{\text {const }}=\dot{Q} / A_{H P}=V_{D C} I_{D C} / A_{H P}$, where $\dot{Q}$ is the heating plate heat flux, $V_{D C}$ and $I_{D C}$ are the respective heating wire electrical DC voltage and current, and $A_{H P}$ is the heating plate heating surface. The effect of assuming $\dot{q}_{\text {const }}$ for $\dot{q}$ in Eq. 3 instead of $\dot{q}_{\text {local }}$ is discussed in Sect. 5.5.

\subsubsection{Calibration of the test section thermocouples}

An advantage of liquid metal facilities is that, at sufficiently high mass flow rate, one can assume a constant fluid temperature over a section of the facility. In this case, the test section. Under isothermal conditions, taking the maximum possible flow rate of the facility and estimating the thermal losses of the test section (Stephan et al. 2019), the fluid temperature in the test section is calculated to be theoretically constant within $\pm 0.05^{\circ} \mathrm{C}$. Under these conditions, the thermocouples of all probes and those of the heating plate were in situ calibrated relative to a master thermocouple (TC3 of $P 1$ in Fig. 1). This was done for 11 temperature levels within a representative temperature range for the experiment. An average offset value over this temperature range 
for each thermocouple relative to the master thermocouple was then calculated. After offset correcting the temperature readings, we could observe that the thermocouple readings of all thermocouples in the test section were precise within $\pm 0.31^{\circ} \mathrm{C}$ relative to the master thermocouple $(95.45 \%$ confidence interval). Between the same master thermocouple and all the heating plate thermocouples, the precision improves to $\pm 0.03^{\circ} \mathrm{C}$ (95.45\% confidence interval). These worst-case value was repeatable on different days. However, the trueness of the temperature measurements is still within the typical uncertainty for thermocouples.

\subsubsection{Energy balance of the facility}

The energy balance of the facility is monitored by comparing the electrical heat input versus the temperature difference between the inlet and outlet of the facility heat exchanger. Due to the relatively high mass flows and relatively low heating plate power input, the measured temperature differences between the heat exchanger inlet and outlet cannot be distinguished from their experimental uncertainty with sufficient precision. Hence, the facility energy balance could not be accurately assessed to the required precision. However, after performing a simple sensitivity analysis based on rough estimations of the experiment thermal losses, the experiment non-dimensional parameters did not show important variations to the values indicated in this publication. Nevertheless, this important issue definitely needs to and is going to be addressed in future experiments by taking respective technical measures.

\section{Instrumentation for the local measurement of the fluid velocity and temperature}

\subsection{The permanent magnet probe}

For this experiment, permanent magnet probes (PMP) were developed to simultaneously measure the local temperature and velocity profiles. Each used probe is named from now on as indicated in Fig. 1. Their position in the test section scaled by the step height is depicted in Fig. 2. The reason for selecting this kind of measurement technique instead of other alternatives is discussed in detail in Schaub et al. (2021), as well as the probe and measurement chain design, the calibration methodology and its results.

A picture of a probe mounted into its housing as they are used in the experiment is shown in Fig. 4, while a sketch is shown in Fig. 5. The probe consists basically of a ringshaped permanent magnet with radial magnetization direction and three type $K$ thermocouples. If a permanent magnet with a magnetic field $B$ is placed in a liquid metal flow with local velocity $\vec{u}$, an electro-motive force (EMF) is induced according to Faraday's law of induction. If two electrodes are placed within the induced electric field $\underset{\text { ind }}{\vec{E}}$, a potential $\nabla \phi=\underset{\text { ind }}{\vec{E}}$ can be measured. If two thermocouples (TC1 and $T C 2$ ) are used instead of two regular copper wires, the fluid velocity-proportional signal and the local fluid temperature can be measured. The temperature measurement is also required for a temperature-correction of the velocity signal. A third thermocouple (TC3) is passed through the permanent magnet to measure the local fluid temperature. Also, the signal amplification ground level is taken over the sheath of TC3. All components are mounted into a PEEK component

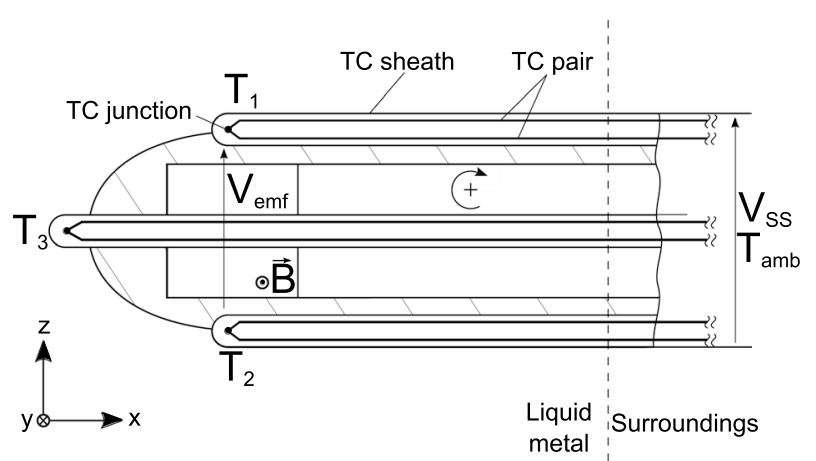

Fig. 5 Sketch of a permanent magnet probe
Fig. 4 Picture of a permanent magnet probe. Probe tip diameter $d_{t i p}=1.6(\mathrm{~mm})$. The permanent magnet is embedded in the probe tip

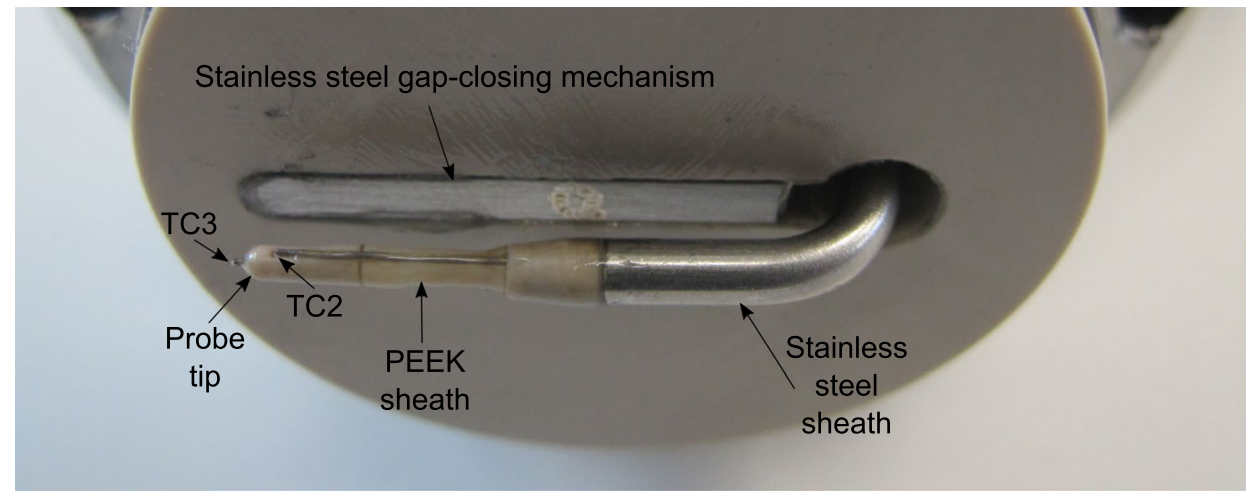


holder. Thermocouples $T C 1$ and $T C 2$ are guided outside of the stainless steel probe holder to the probe tip along a nut carefully milled on the PEEK sheath.

For the probes, ungrounded thermocouples are used (Fig. 5) to simplify the temperature-correction procedure to account for further induced thermoelectric effects. By manufacturing the probe tip of an insulating material and ensuring that all components are electrically insulated from each other across the probe tip, further simplifications can be made. Thermocouples $T C 1$ and $T C 2$ are electrically insulated from the liquid metal with glue along their extension except for the immediate region of tips. Inside the stainless steel probe sheath, all three thermocouples are electrically insulated from each other.

The resulting measurement equation reads.

$V_{S S}=\mathrm{Ku}_{\mathrm{vol}}+S_{e f f}\left(T_{2}-T_{1}\right)+V_{0}$

where $V_{S S}$ is the measured voltage over the thermocouple sheaths, $\mathrm{K}$ is the calibration constant of the probe to be determined experimentally, $\mathrm{u}_{\mathrm{vol}}$ is the liquid metal volumeaveraged flow velocity near the probe, $S_{\text {eff }}=\left(S_{S S}-S_{G a I n S n}\right)$ can be interpreted as an effective Seebeck coefficient for the probe, where $S_{S S}$ and $S_{G a I n S n}$ are the Seebeck coefficients of stainless steel (SS) and GaInSn, $T_{2}$ and $T_{1}$ are the temperatures measured by thermocouples $T C 2$ and $T C 1$, and $V_{0}$ is the offset of the measurement.

The probes are traversed along the $y$-axis with linear actuators. To avoid any probe tip damage while driving the probes to the $y$-direction range end, a mechanical blocking system was built into the probe housing (not seen in the figures). Since measurements are distorted near the wall region due to the presence of the probe, measurements are take keeping a distance of $5 \mathrm{~mm}$ to the walls. The six probes ( $P 1$ to $P 6$ in Fig. 1) are sequentially used to measure velocity profiles along the test section. A probe-parking-and-gap-closing mechanism was developed to avoid any flow disturbance by upstream probes. When a probe is in use, the mechanism closes the parking gap left by the probe (Fig. 4). When approaching the probe to the test section walls, we observed non-negligible flow disturbances due to the presence of the invasive probe.

\subsection{Permanent magnet probe measurement chain}

The six velocity-proportional induced signals $V_{S S}$ are first amplified, then digitized, noise-shaped and decimated to a PC with an 8-channel LTT24 measurement system from Labortechnik Tasler GmbH. The induced signals $V_{S S}$ are in the order of a few microvolts $(\mu V)$, i.e., a special strategy to suppress any external electromagnetic interference with the signals was developed. These interferences are managed to be suppressed down to $\sim 80 \mathrm{nV}$ relative to the intrinsic physical noise contributions of any measurement device (flicker and thermal noise). This value is representative for all probes and under realistic experimental conditions. More details are discussed in in Schaub et al. (2021). The probe thermocouples (as well as the heating plate ones) are connected to a cold junction (ISOTECH TRU937) and then to a National Instruments data acquisition system.

\subsection{Measurement capabilities of the present probe experimental setup}

In this paper, only first moment statistics for the probe velocity-proportional signal are presented. The measurement of the velocity variance $u^{\prime 2}$, the time-averaged temperature $T$, the temperature variance $T^{\prime 2}$ and the turbulent heat flux $u^{\prime} T^{\prime}$ is indeed possible with the present probe design and measurement system. However, further development of the measurement equation, an optimization of the facility temperature inlet boundary condition (Sect. 2.3.1) and some minor rewiring of the measurement chain are required.

Equation 4 is the measurement equation for $V_{S S}=V_{S S}(t)$, i.e., it is an instant quantity. The calculation of first moment statistics from Eq. 4 is straightforward, since all required mathematical operations to be applied on Eq. 4 are linear. For higher moment statistics though, Eq. 4 needs to be extended (Kapulla (2000)). Particularly, to properly temperature compensate the measured signal for the measurement of the velocity variance $u^{\prime 2}$, the temperature-correction term must be corrected for dynamic effects, since the temperature measurement is not in phase with the velocity measurement. Kapulla (2000) addresses this issue with a dynamic thermocouple model and includes it into Eq. 4. The main advantage of permanent magnet probes compared to other usual measurement techniques for liquid metal flows relies on the fact that they are able to measure locally and simultaneously both the flow velocity and its temperature with a good frequency response.

Since the velocity and the temperature signals of the probe are sampled by different measurement systems (Sect. 3.2), at this stage of the project, it is not possible to measure $u^{\prime} T^{\prime}$. In future experiments, either both systems will be synchronized or the thermocouple signals of one probe will be connected to the LLT24 measurement system to its two available channels. For the latter, rewiring of the probe is necessary, being this the reason why this was not done for the present experimental campaign. 


\section{Covered parameter set and general remarks on the experiment}

\subsection{Parameter set}

The facility operational ranges are shown in Table 2, where $T_{\text {ref }}$ is the test section inlet temperature (dependent on the facility heat input), $\dot{V}$ is the volume flow rate, $\dot{m}$ the mass flow rate, $\dot{q}$ is the heating plate heat flux input, $R e_{h}$ is the Reynolds number (to be defined below, see Eq. 5), Pr is the Prandl number evaluated in the $T_{r e f}$ range, $P e_{h}$ is the Peclet number taking $\operatorname{Pr}$ at $25^{\circ} \mathrm{C}$ and varying $R e_{h}$ over its range, and $R i_{h}$ is the Richardson number (to be defined below, see Eq. 6) varying $R e_{h}$ over its range and taking $\dot{q}$ at its maximum value and the fluid properties at $25^{\circ} \mathrm{C}$. For the experiments, $T_{\text {ref }}=T_{\text {inlet }}=25^{\circ} \mathrm{C}$. It is important to remark the difference between the possible facility operational ranges from the possible experimental operational range. The latter is limited by the instrumentation.

The covered experimental parameter range in terms of the Reynolds and Richardson numbers is listed in Table 3, where the Reynolds number is defined as.

Table 2 Facility operational range for different parameters

\begin{tabular}{lll}
\hline & Minimum value & Maximum value \\
\hline$T_{\text {ref }}=T_{\text {inlet }}\left({ }^{\circ} \mathrm{C}\right)$ & 15 & 32 \\
$\dot{V}(\mathrm{l} / \mathrm{s})$ & 0.1 & 2.0 \\
$U_{\text {bulk,inlet }}(\mathrm{m} / \mathrm{s})$ & 0.031 & \\
$U_{\text {bulk, outlet }}(\mathrm{m} / \mathrm{s})$ & 0.016 & 0.625 \\
$\dot{m}(\mathrm{~kg} / \mathrm{s})$ & 0.64 & 0.313 \\
$\dot{q}\left(\mathrm{~W} / \mathrm{m}^{2}\right)$ & - & 12.82 \\
$R e_{h}$ & & \\
$P r$ & 3813 & 14985 \\
$P e_{h}$ & 0.036 & 76256 \\
$R i_{h}$ & 128 & 0.032 \\
\hline
\end{tabular}

$R e_{h}=\frac{U_{b} h}{v_{r e f}}$

where $U_{b}$ is the inlet bulk velocity, $h$ is the step height, and $v_{r e f}$ is the kinematic viscosity of GaInSn at the reference temperature $T_{\text {ref }}=25^{\circ} \mathrm{C}$.

The Richardson number is calculated as usually for this case as.

$R i_{h}=\frac{g \beta \Delta T^{*} h}{U_{b}^{2}}$, with $\Delta T^{*}=\frac{\dot{q} h}{\lambda}$,

where $g$ is the gravitational acceleration, $\beta$ the volumetric expansion, $\Delta T^{*}$ the characteristic temperature difference between the heating plate and bulk flow due to the input heat flux $\dot{q}, \lambda_{\text {ref }}$ the thermal conductivity of the working fluid at the reference temperature, $h$ the step's height, and $U_{b}$ the velocity of the bulk flow at the inlet.

The idea behind the definition of the parameter set was to cover both forced- and mixed convection regimes at sufficiently high Reynolds numbers. That is, for Reynolds numbers where the BFS flow can be considered as fully turbulent and stagnated, i.e., with a constant mean reattachment length $x_{R}$. According to Nadge and Govardhan (2014), this should be the case for BFS flows with an $E R>1.8$ and $R e_{h} \geq 20000$. The aim of including $R e_{h}=10000$ was to observe the BFS stagnation process.

Lloyd and Sparrow (1970) predicted analytically the critical Richardson number for the onset of buoyancy effects for a vertical flat plate for various Prandtl number $(P r)$ fluids. They predicted a critical Richardson number $R i_{h, t h} \approx 0.05$ for $0.003<\operatorname{Pr}<0.03$. In the absence of alternatives at the time of planning the experiment, this value was taken as a rough order of magnitude estimate for the onset of mixed convection, well aware of the differences between a vertical flat plate and a vertical confined BFS. As will be shown later, this estimate seems to underpredict the measured onset of mixed convection for the present experiment by one order of magnitude. Thus, a more representative order of magnitude estimate for the critical Richardson number was derived afterwards for the actual present flow by means

Table 3 Covered parameter set

\begin{tabular}{lllllll}
\hline$\dot{V}(l / s)$ & $\dot{m}(\mathrm{~kg} / \mathrm{s}$ & $\dot{Q}(W)$ & $\dot{q}\left(W / \mathrm{m}^{2}\right)$ & $R e_{h}$ & $\begin{array}{l}R e_{h} \text { (approxi- } \\
\text { mated })\end{array}$ & $R i_{h}$ \\
\hline 0.267 & 1.71 & 28 & 585 & 10180 & 10000 & 0.006 \\
0.530 & 3.40 & 108 & 2245 & 20208 & 20000 & \\
0.792 & 5.08 & 232 & 4841 & 30197 & 30000 & \\
0.266 & 3.40 & 184 & 3824 & 10142 & 10000 & 0.04 \\
0.268 & 1.71 & 685 & 14274 & 10218 & & 0.15 \\
0.530 & 3.40 & 179 & 3729 & 20208 & 20000 & 0.01 \\
0.529 & 3.39 & 703 & 14644 & 20170 & & 0.04 \\
\hline
\end{tabular}


of scale analysis based on the work of Li and Djilali (1995) (see Sect. 6).

\subsection{General remarks}

\subsubsection{Measurement uncertainty and data visualization}

The detailed uncertainty analysis methodology of the presented data is presented in the appendix. The uncertainty estimation was calculated following the methodology proposed by the Guide to the Expression of Uncertainty in Measurement (GUM) from the Joint Committee for Guides in Metrology (JCGM). All shown error bars are expressed as combined extended uncertainties for a $95.45 \%$ confidence interval.

The measured profiles for each probe in the next section are shown following the nomenclature of Fig. 1. The exact position of each probe can be read out of Fig. 2. The error bars are shown only for those probes, for which the error bar is clearly distinguishable from the respective data symbol. For the sake of a better visualization of some profiles, only selected - yet generally representative-error bars are shown.

The measured probe sensitivities for each probe and its respective extended measurement uncertainty are presented in Table 4. The difference between the probes in the measured sensitivities and their respective extended combined uncertainty is explained by typical issues regarding the wetting of surfaces with GaInSn. These aspects are discussed in detail in Schaub et al. (2021).

For the local Nusselt number along the heating plate, some data points are missing since for these points the respective thermocouples broke during the mounting of the experiment.

\subsubsection{Experiment conduction}

During the measurement campaign, the liquid metal is not drained from the facility. Before the measurement of every parameter set, the instrumentation is warmed up for at least

Table 4 Measured probe sensitivities and extended combined uncertainty

\begin{tabular}{lll}
\hline & Sensitivity $(\mu \mathrm{V} / \mathrm{m} / \mathrm{s})$ & $\begin{array}{l}\text { Extendedcombined } \\
\text { uncertainty }(\mu \mathrm{V} / \mathrm{m} / \mathrm{s})\end{array}$ \\
\hline P1 & 52.41 & 1.72 \\
P2 & 50.85 & 1.16 \\
P3 & 41.95 & 6.42 \\
P4 & 23.18 & 8.50 \\
P5 & 43.97 & 2.91 \\
P6 & 54.82 & 3.99 \\
\hline
\end{tabular}

12 hours and the facility was let to thermally stabilize for at least 2 hours. The thermal steady state is judged by monitoring all thermocouple readings to be stable in time for at least 30 minutes. During the measurements, all thermocouple readings are monitored and were observed to lay within the mentioned variation levels in Sect. 2.3.1.

The data were logged in two consecutive series or runs. In the first run, the velocity profiles were measured with the probes. During each profile measurement, all probes but the one being used were parked. Once all six velocity profiles were measured, the heating plate temperatures were measured simultaneously in a second run right after finishing the first run. For the second run, all probes were parked, but $P 1$ to measure the flow inlet temperature. $P 1$ was positioned $5 \mathrm{~mm}$ from its parking position to reduce its influence on the flow down to a minimum. The facility temperature variability was zero-averaged by measuring the heating plate temperatures over five full temperature variability periods.

\subsubsection{Sampling rate and sampling time}

The sampling time per velocity measurement point and the respective sampling rate were calculated based on the statistical analysis presented by Nobach and Tropea (2007) and taking into consideration the optimum operation of the LTT24 measurement system.

The chosen sampling time per velocity measurement point was $t_{s}=105 \mathrm{~s}$ at a decimation rate (equivalent to an analog sampling rate) of $f_{d}=5000 \mathrm{~Hz}$ and using a 8pole Butterworth low-pass filter with cutoff frequency $f_{c}=200 \mathrm{~Hz}$. Of course, for the measurement of mean quantities, a much lower sampling rate would have been sufficient. However, as already mentioned, an optimum operation of the LTT24 measurement system had to be guaranteed; hence, a higher decimation rate was chosen than actually needed. The chosen cutoff frequency $f_{c}$ was calculated taking the probe diameter $d_{\text {tip }}=1.6 \mathrm{~mm}$ and the maximum bulk inlet velocity for the measurement campaign $U_{b}=0.247 \mathrm{~m} / \mathrm{s}$, i.e., the probe frequency cutoff is $f_{\text {tip }}=154 \mathrm{~Hz}$.

For the measurement of the heating plate temperatures, which we recall were measured in a separate run after the measurement run of the velocity profiles, the sampling time $T_{s}$ was between $845 s<T_{s}<1779 s$, corresponding to five facility temperature variability periods.

\section{Experimental results}

\subsection{Constant $R i_{h}=0.006$, variable $R e_{h}$}

The measured dimensionless velocity profiles for probes $P 1$ to $P 6$ (Fig. 1) and the local Nusselt number along the heating plate for this parameter set are shown in Fig. 6 and Fig. 7, 
respectively. The local Nusselt number is shown for the thermocouple center column, i.e., column $C$ in Fig. 2. The error bars of the velocity profile data are depicted for $R e_{h} \approx 30000$ and only for $P 3$ and $P 4$.

As intended from design for calibration purposes, the flow enters the test section in a flat profile $(P 1)$. From $P 2$, one can observe a defined boundary layer which can be assumed to be turbulent due to the trip wire. Since the used probes are invasive, the velocity data for $P 3$ near and in the recirculation zone of the BFS has a high uncertainty which is not considered in the error bars. The mean reattachment point $x_{R}$ is between $P 3$ and $P 4$, i.e., $5 h<x_{R}<10 h$ (Fig. 2). After $x_{R}$, the flow returns slowly to equilibrium. The velocity profiles for all $R e_{h}$ agree with each other within experimental uncertainty. The general flow structure seems to be independent on $R e_{h}$; however, slightly higher non-dimensional velocities are observed for the higher velocity regions for $R e_{h} \sim 30000$. This can be explained in terms of local fluid dynamical phenomena around the probe tip, as explained in detail in Schaub et al. (2021). In general terms, the higher the fluid velocity, the thinner the fluid velocity boundary layer and hence the higher the volume-averaged fluid velocity around the probe tip. In other words, this nonlinear interaction between the fluid flow at higher velocities and the measured probe sensitivities may explain the slightly higher measured values for $R e_{h} \sim 30000$.

For $P r<1$ fluids, $N u$ is relatively low, meaning that the convective and conductive heat transfer rates are comparable in magnitude to each other (in order of magnitude sense). For this reason, the higher $R e_{h}$, the higher $N u$, as can be seen in Fig. 7. For the shown cases, i.e., forced convection cases, temperature is transported as a passive scalar, i.e., the BFS flow is not expected to exhibit alterations from its typical qualitative behavior, as can be concluded from the velocity profiles.

For all studied $R e_{h}$, a $N u$ maxima can be estimated at $x \approx 8.75 h$. This means that at this point $\Delta T$ is lowest. As will be shown in Sect. 5.5, this does not necessarily mean that the mean reattachment point $x_{R}$ is also located at this point, since thermal conduction within the heating plate must be taken into consideration (conjugate heat transfer problem.

Besides the main maxima at $x \approx 8.75$, two further maxima can be observed at $x \approx 5 h$ and $x \approx 12.5 h$. One may argue that expected shape for the local $\mathrm{Nu}$, i.e., a nearly linear increase/decrease around $x_{R}$, may be contained within the error bars. However, the maxima appear in all measured cases; they pronounce with increasing $R e_{h}$ and seem to be independent on the heat input. After inspecting pictures made of the heating plate during its manufacturing and mounting on the test section, no evidence was found on possible thermal boundary condition changes during the experiment. However, we cannot fully discard that these two maxima are an artifact of our experimental setup. A further possible explanation is that they arise as a result of the influence of secondary motions of the second kind. However, it is hard to test these hypotheses without any further data. The only available DNS data comparable to this experiment are that of Oder et al. (2019), which does not contain the local Nusselt number distribution along the heating plate.

\subsection{Variable $R i_{h}$, constant $R e_{h} \approx 10,000$}

The measured dimensionless velocity profiles and Nusselt number distribution are shown in Fig. 8 and Fig. 9, respectively. The Nusselt number distribution is shown for the thermocouple center column, i.e., column $C$ in Fig. 2. The error bars of the velocity profile data are depicted for $R i_{h}=0.15$ and for all probes but $P 1$ and $P 2$.

This parameter set shows the same behavior for $P 1$ and $P 2$. As mentioned in Sect. 4.1, an onset of buoyancy effects on the flow was expected at $R i_{h, t h} \sim 0.05$, i.e., for the $R i_{h}=0.04$ case. When looking at Fig. 8 , the velocity profiles for $R i_{h}=0.04$ and $R i_{h}=0.006$ coincide within experimental uncertainty. Although the error bars for $R i_{h}=0.15$ overlap with those for the other cases in some extent, a slight deviation is observed. This tendency is explained by the fluid acceleration near the heating plate due to buoyancy forces opposing to the fluid motion in the recirculating region. This behavior is characteristic to the mixed convection regime for a BFS and is also observed in DNS simulations (Nieman and Fröhlich 2016). This indicates that our first order of magnitude estimate for $R i_{h, c r i t}$ underestimates the mixed convection onset by about one order of magnitude. This is not a surprise, since the mentioned thresholds were calculated for a vertical flat plate (Lloyd and Sparrow (1970)).

The measured mixed convection onset at $R i_{h}=0.15$ is almost not observable in the $N u$ distributions. All values but the first one at $x \approx 0.5 h$ cannot be distinguished from each other within experimental uncertainty. The reason for this is explained in Sect. 5.5.

\subsection{Variable $R i_{h}$, constant $R e_{h} \approx 20,000$}

The measured dimensionless velocity profiles and Nusselt number distribution for this parameter set are illustrated in Fig. 10 and Fig. 11, respectively. The Nusselt number distribution is shown for the thermocouple center column, i.e., column $C$ in Fig. 2. The error bars of the velocity profile data are depicted for $R i_{h}=0.04$ and only for probes $P 3$ and $P 4$.

The observations made for the $R e_{h} \approx 10000$ and variable $R i_{h}$ case can be transferred to this case. It seems that the onset of mixed convection is independent $R e_{h}$, although this hypothesis could not be validated in the present experiment. For $R e_{h} \approx 20000$ and $R i_{h}=0.04$, the heating plate reached 


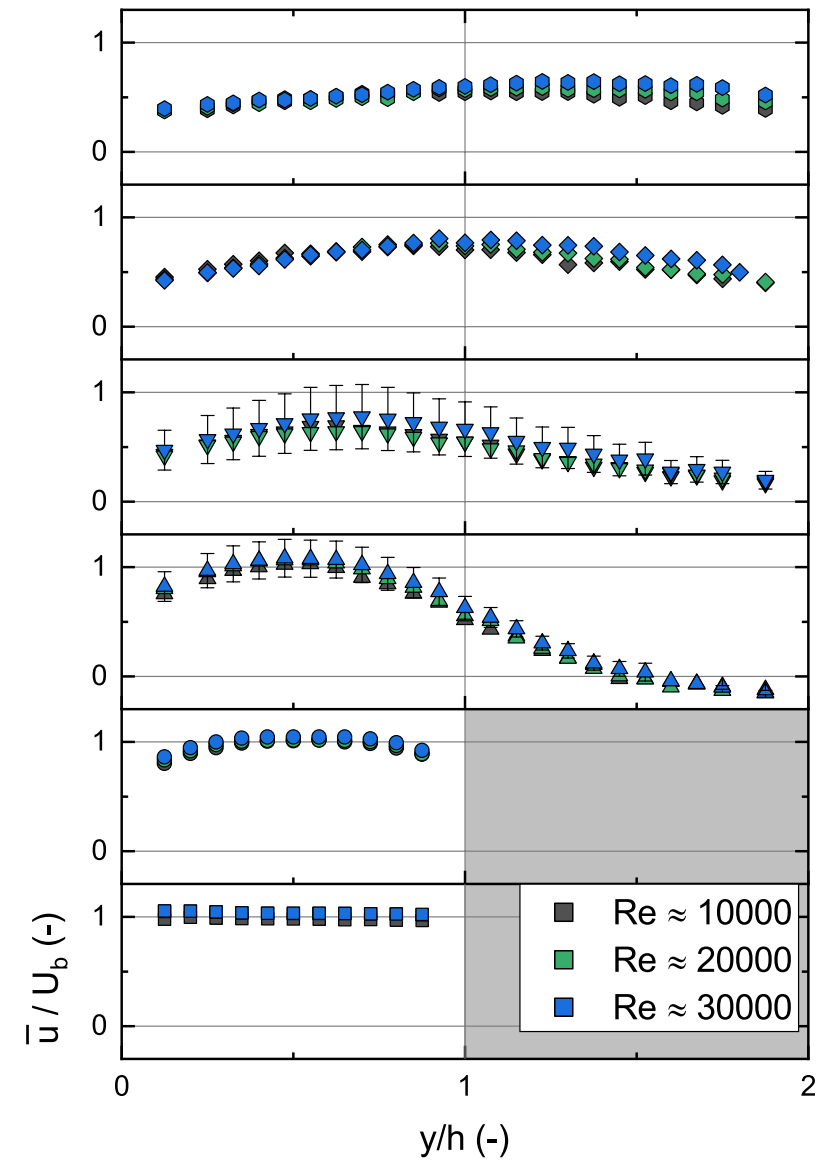

Fig. 6 Measured dimensionless velocity profiles for a constant $R i=0.006$ and variable $R e_{h}$ at different positions (see Fig. 2 for the exact positions)

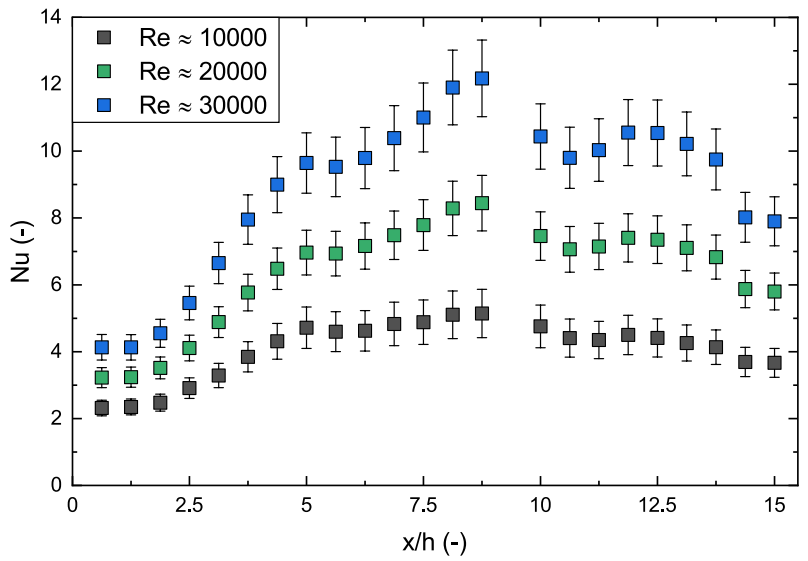

Fig. 7 Measured local Nusselt number on the heating plate centerplane fo $R i=0.006$ and variable $R e_{h}$

its upper operation limit; hence, the $R i_{h}=0.15$ case could not be measured.

\subsection{Symmetry of $\mathrm{Nu}$ for $\mathrm{Re}_{\mathrm{h}} \approx 20208, \mathrm{Ri}_{\mathrm{h}}=0.04$}

In the context of a confined BFS experiment, the question regarding the flow symmetry must be addressed. As shown in Fig. 2, a total of five thermocouple columns were integrated into the heating plate. The idea was to obtain a detailed panorama of the local $N u$ distribution on the heating plate. The result of columns $A(z \approx 0.66 h(-)), C(z=0 h(-))$ and $E(z=0.66 h(-))$ is shown in Fig. 12. All error bars are shown. A point to consider is that the actual temperature differences between the columns are below $0.8^{\circ} \mathrm{C}$ for the highest heat rates and even lower for the rest of the cases.

The recorded local $N u$ distribution is not symmetric. Although the error bars of columns $C$ and $E$ overlap, a lower trend for that the data points for column $A$ is observed. Particularly in the region where the $N u$ maxima are located, i.e., the region where the minima for $\Delta T=T_{w}-T_{b}$ are located.

The most likely explanation for the $N u$ asymmetry is the construction of the heating plate itself and not an actual flow asymmetry. In Fig. 13, the exterior side (back-side) of the inner copper block is shown. This is also the side from which the thermocouples are inserted into the block. All thermocouples are guided from one side, making the thermal path resistance on this side higher. That is, heat tends to diffuse into the flow on the side of column $A$, inducing the highest $\Delta T$ and hence the lowest $N u$. The fact that the region near the is $N u$ maxima at $x / h \approx 8.75(-)$ is where the three curves separate the most, strengthens our argument.

\subsection{Why can't the mixed convection onset be observed in the $\mathrm{Nu}$ profiles yet in the velocity profiles it can?}

To answer this question, we ran conjugate heat transfer numerical simulations of the experiment. The Reynoldsaveraged Navier-Stokes (RANS) equations, including the heating plate as a solid body, were solved for the test section. A baseline Reynolds Stress model was used for the calculation of the Reynolds stress tensor, while the Kays correlation was used for the calculation of the turbulent Prandtl number. Further setup details of the simulation are explained in the appendix (Sect. 11).

This section is not intended to be a validation of our numerical simulations, but to offer a qualitative comparison between our numerical and experimental results. We only aim to show the qualitative effect that the way the specific heat flux $\dot{q}$ is calculated has on the local $N u$ distribution along the heating plate.

In Fig. 14, we show the numerical results for the local $\mathrm{Nu}$ distribution along the heating plate center-plane for $R i_{h}=0.006$ and $R i_{h}=0.15$ at $R e_{h}=10218$ together with our experimental results for $N u$ for $R i_{h}=0.15$ at $R e_{h} \approx 10000$ (same data points as for Fig. 8). Four curves for the 
numerical results of $\mathrm{Nu}$ are depicted in Fig. 14, two for each $R i_{h}$. The difference between the two curves for each dimensionless parameter set relies only on what $\dot{q}$ was used to calculate $N u$ (Eq. 3): once assuming a constant heat flux over the heating plate, i.e., $\dot{q}=\dot{Q} / A_{H P}=\dot{q}_{\text {const }}$ (as for the experiment), and once using the local specific heat flux distribution along the heating plate as obtained from the numerical results, i.e., $\dot{q}=\dot{q}_{\text {local }}=\lambda_{\text {fluid }} \partial T / \partial y$, where $\partial T / \partial y$ is evaluated locally at the heated wall surface.

The heating plate Biot number is $B i=O\left(10^{-1}\right)$. Although for $B i=O\left(10^{-1}\right)$ the $B i \ll 1$ condition is not fully met, at first, one may take a constant heat flux boundary condition as a first approximation. In fact, in Fig. 14 we observe that the qualitative resemblance in trend between the experimental and numerical results for the $R i_{h}=0.15$ and $\dot{q}_{\text {const }}$ case is good. Even the $N u$ trend at lower $x / h$ in Fig. 9 is captured well by the numerical results. However, the disagreement between the $\dot{q}_{\text {const }}$ and $\dot{q}_{\text {local }}$ cases in Fig. 14 is clear. This evidences that a constant specific heat flux boundary condition is not applicable to this case. The correct thermal boundary condition for the heating plate is a mixed-type boundary

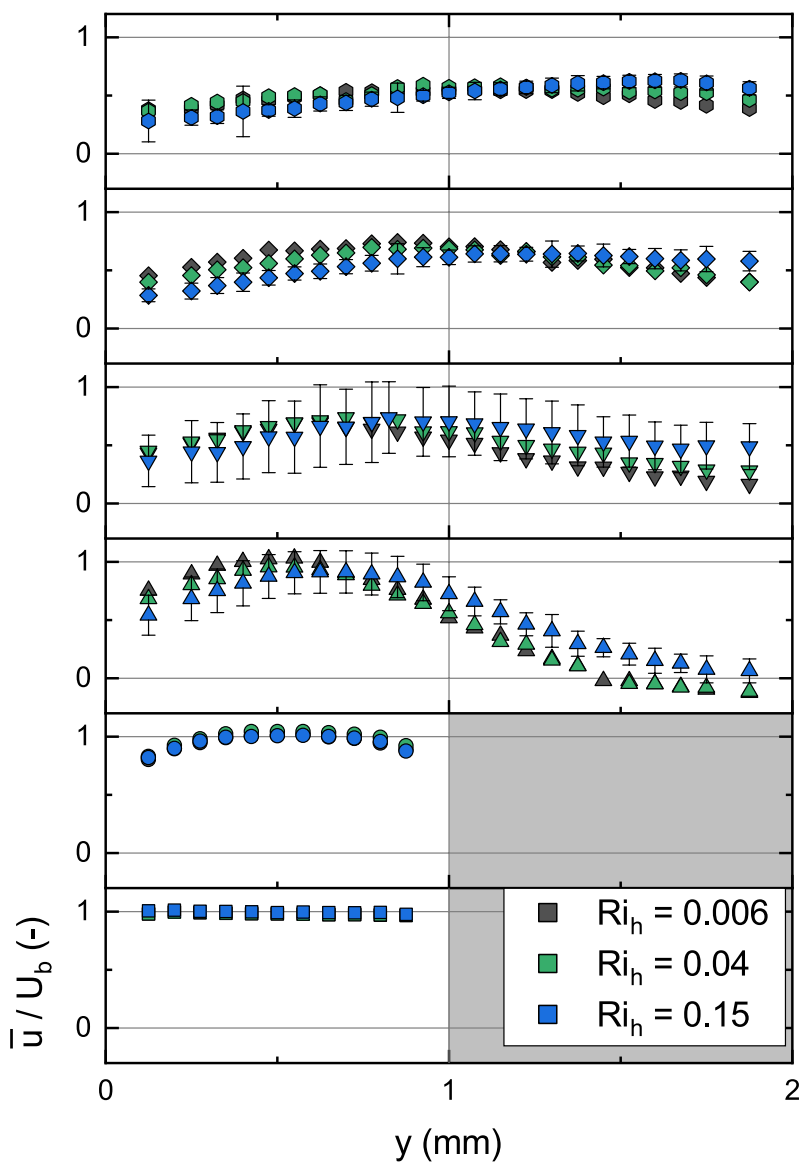

Fig. 8 Measured dimensionless velocity profiles for a constant $R e_{h} \approx 10000$ and variable $R i_{h}$ at different positions

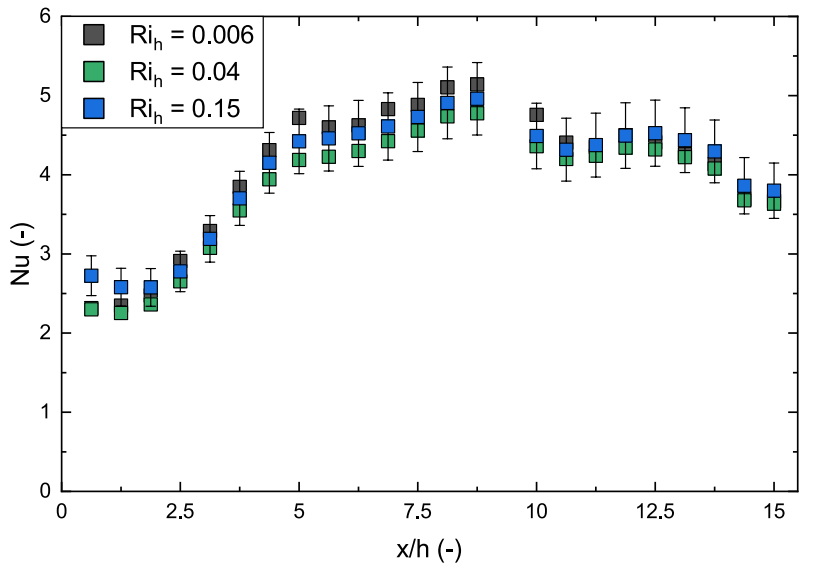

Fig. 9 Measured local Nusselt number on the heating plate centerplane for $R e_{h} \approx 10000$ and variable $R i_{h}$

condition. $N u$ is hence a function of both the local $\Delta T(x, z)$ and $\dot{q}(x, z)$. The local dependency of $\dot{q}$ and $\Delta T$ explains why only for the $\dot{q}_{\text {local }}$ cases in Fig. 14 the mixed convection case can be distinguished from the forced convection case. In other words, the reason why we could not observe the onset to mixed convection in Fig. 9 is explained by the way we defined $N u$, i.e., by taking $\dot{q}=\dot{q}_{\text {const }}$ in Eq. 3. This high sensitivity on thermal boundary condition is typical for liquid metal flows (Straub et al. (2019) and references therein).

To measure $\dot{q}_{\text {local }}$ is not a trivial task. As mentioned in Sect. 2.3.3, an alternative to the measurement of $\dot{q}_{\text {local }}$ is to locally measure $T$ and then solve the two-dimensional inverse Fourier problem as proposed by Woodfield et al. (2006). This procedure will be implemented in future experiments.

It is important to assess the physical interpretation to be given to $\mathrm{Nu}$ in Fig. 14 . For the $\dot{q}_{\text {const }}$ cases, $N u$ may be interpreted as the reciprocate of the dimensionless wall temperature only. For the $\dot{q}_{\text {local }}$ cases though, $N u$ can be interpreted approximately proportional to the dimensionless wall temperature gradient. To interpret $N u$ as usual, i.e., as the actual dimensionless wall temperature gradient, $T_{b u l k}$ needs to be determined ad hoc (Moffat (1998), Weigand (2015)). We defined $T_{b}=T_{\text {inlet }}$ in the absence of other alternatives.

In Fig. 15, we show the streamwise component skin friction coefficient $C_{f}$ along the heating plate center-plane, where $C_{f}=\tau_{w} / \rho U_{b}^{2}$ with $\tau_{w}$ the wall shear stress. Flow stagnation regions can be identified by zero-crossings of $C_{f}$. The general behavior of $C_{f}$ coincides with that reported in Niemann \& Fröhlich (2016a, b). It is interesting to note that for this case, contrary to the mentioned DNS simulations, the flow reattachment point $x_{R}$, defined as $C_{f}$ zero-crossings at $x / h \approx 10$ and $x / h \approx 5$ for $R i_{h}=0.006$ and $R i_{h}=0.15$ respectively, cannot be related in any obvious way to $\mathrm{Nu}$ in Fig. 14. This means that for the case of a mixed thermal 


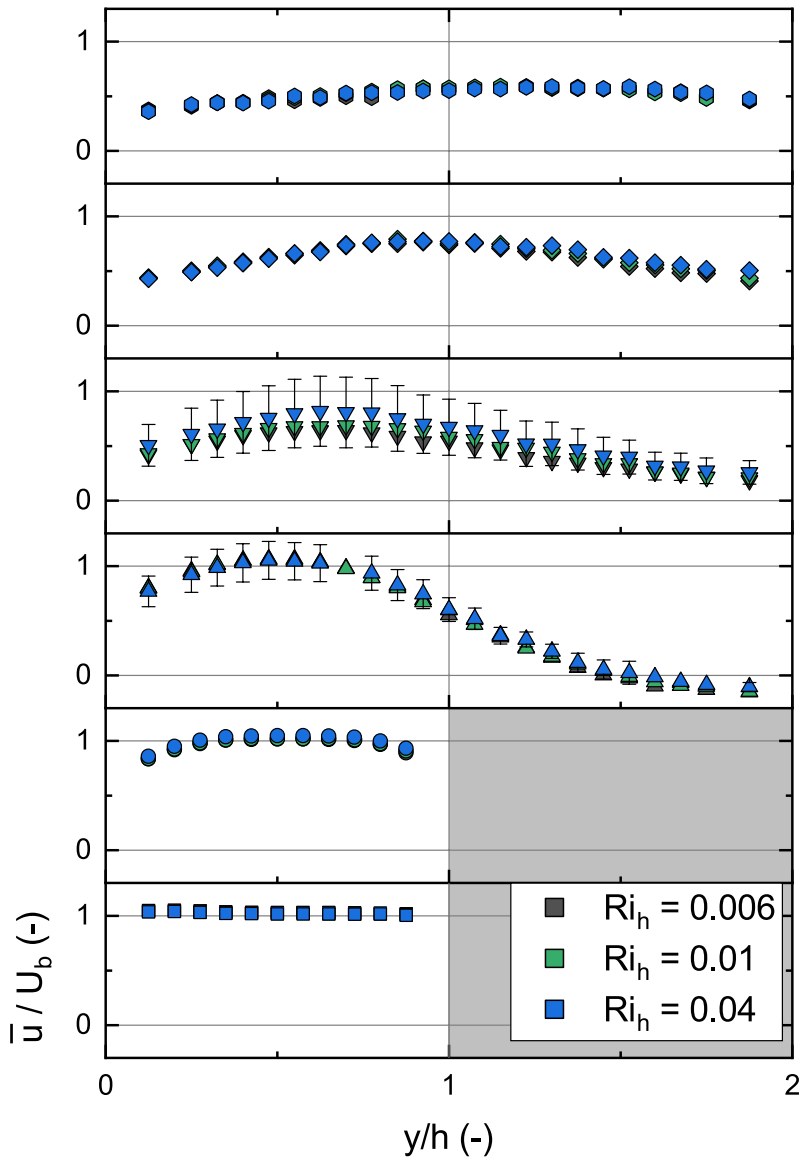

Fig. 10 Measured dimensionless velocity profiles for a $R e_{h} \approx 20000$ and variable $R i_{h}$ at different positions

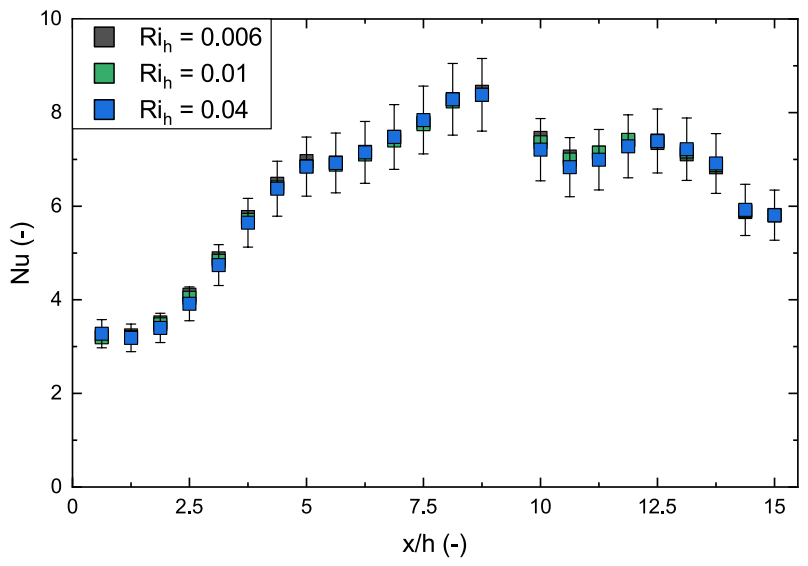

Fig. 11 Measured local Nusselt number on the heating plate centerplane for $R e_{h} \approx 20000$ and variable $R i_{h}$

boundary condition $\mathrm{Nu}$ loses its capability of being an indicator of $x_{R}$. This probably is explained by complex threedimensional flow characteristic of a confined BFS together

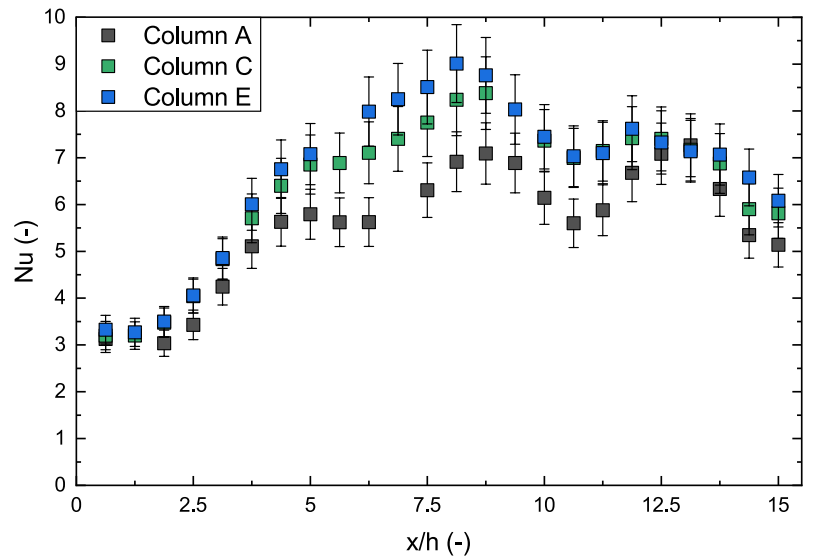

Fig. 12 Local Nusselt for three thermocouple columns on the heating plate for $R e_{h} \approx 20000$ and $R i_{h}=0.04$

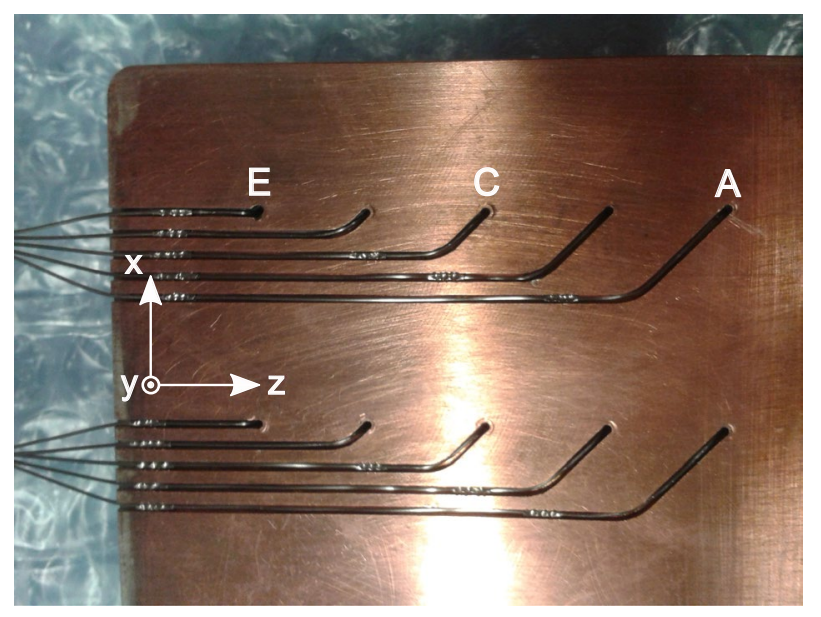

Fig. 13 Picture of the back of the heating plate

with the conjugate heat transfer problem. We expect that the heat transfer contributions of secondary motions of the second kind to play an important role, as they may transport cold incoming fluid along the side walls to the heating plate center-plane (Oder et al. (2019)). In addition, it is to be expected that the heat transfer within the heating plate plays an important role. As a final remark, looking at Fig. 8, the experiment $x_{R}$ seems to be indeed in the region predicted by our numerical simulations. However, to gain a more comprehensive picture of the flow physics, high fidelity conjugate heat transfer numerical simulations of the experiment are necessary. 


\section{Critical Richardson number order of magnitude estimate}

The following analysis is based on Li and Djilali (1995) who predicted the $R e_{h}$ qualitative dependency of $x_{R}$ for a non-confined BFS by means of dimensional analysis. Here, a similar scale analysis is presented considering buoyancy effects but neglecting the viscous terms.

The starting point are the $2 D$ Navier-Stokes equations including buoyancy effects, which are modeled assuming the Boussinesq approximation. The steady-state equations read,

$u \frac{\partial u}{\partial x}+v \frac{\partial u}{\partial y}=-\frac{1}{\rho_{\text {ref }}} \frac{\partial p}{\partial x}+v_{r e f}\left(\frac{\partial^{2} u}{\partial x^{2}}+\frac{\partial^{2} u}{\partial y^{2}}\right)+\left(\rho_{r e f} \beta_{r e f} \Delta T\right) g$

$u \frac{\partial v}{\partial x}+v \frac{\partial v}{\partial y}=-\frac{1}{\rho_{r e f}} \frac{\partial p}{\partial y}+v_{r e f}\left(\frac{\partial^{2} v}{\partial x^{2}}+\frac{\partial^{2} v}{\partial y^{2}}\right)$

For scale analysis, the equations are expressed in terms of characteristic length scales, velocities and temperature differences in the recirculation region. These equations read as

$\frac{u_{c}^{2}}{x_{c}}+\frac{v_{c} u_{c}}{y_{c}} \sim-\frac{1}{\rho_{\text {ref }}} \frac{p_{c}}{x_{c}}+v_{r e f}\left(\frac{u_{c}}{x_{c}^{2}}+\frac{u_{c}}{y_{c}^{2}}\right)+\left(\rho_{\text {ref }} \beta_{r e f} \Delta T_{c}\right) g$

$\frac{u_{c} v_{c}}{x_{c}}+\frac{v_{c}^{2}}{y_{c}} \sim-\frac{1}{\rho_{\text {ref }}} \frac{p_{c}}{y_{c}}+v_{r e f}\left(\frac{v_{c}}{x_{c}^{2}}+\frac{v_{c}}{y_{c}^{2}}\right)$

Replacing $v_{c} \sim u_{c} y_{c} / x_{c}$ (continuity) and after some algebra, one gets,

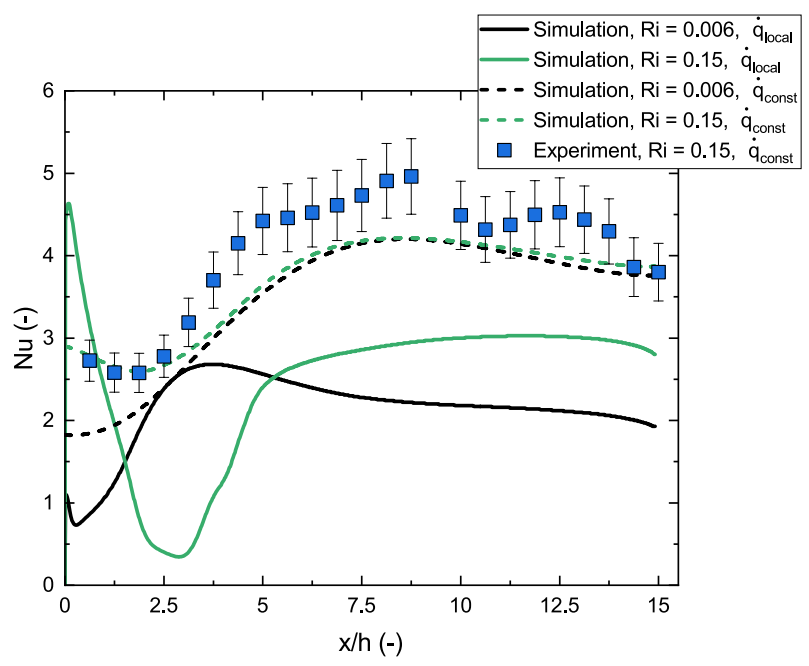

Fig. 14 Qualitative comparison of the local $\mathrm{Nu}$ distribution depending on what $\dot{q}$ is used to calculate $N u$ $u_{c}^{2}\left(\frac{y_{c}^{2}}{x_{c}^{2}}-1\right)+x_{c}\left(\rho_{r e f} \beta_{r e f} \Delta T_{c}\right) g \sim v_{r e f} u_{c} x_{c}\left(\frac{y_{c}^{2}}{x_{c}^{2}}-1\right)\left(\frac{1}{x_{c}^{2}}+\frac{1}{y_{c}^{2}}\right)$

Since this is a rough order of magnitude estimate, the viscous terms are neglected in a next step. After some algebra and rearranging Eq. 11, one gets,

$\frac{y_{c}^{3}}{x_{c}^{3}}-\frac{y_{c}}{x_{c}}-R i_{h} \sim 0$

Considering only the real solutions to Eq. 12 , one obtains $R i_{h}=2 \sqrt{3} / 9 \approx 0.385=O\left(10^{-1}\right)$. Again, this is a rough order of magnitude estimate assuming the case when inertial forces are in the same order as buoyancy forces. However, this result agrees well-in an order of magnitude sensewith the presented measurements. For the case $R e_{h} \approx 10000$ and $R i_{h}=0.15=O\left(10^{-1}\right)$, one can observe in Fig. 8 that buoyancy forces begin to become noticeable.

\section{Conclusions}

We conducted a non-isothermal vertical confined backward facing step experiment with a low-Prandtl fluid. Mean velocity profiles at six streamwise positions along the test section are measured with in-house developed and manufactured permanent magnet probes. The local Nusselt number distribution along the heating plate are measured with thermocouples. Reynolds-averaged Navier-Stokes (RANS) numerical calculations are further performed to assess the influence of the assumed thermal boundary condition for the heating plate.

The main conclusions of the present work are summarized next:

- For the forced convection cases, the velocity profiles are independent on the Reynolds number $10000 \leq R e_{h} \leq 30000$ within experimental uncertainty. However, more data are required to draw more general conclusions.

- The onset of the mixed convection regime is observed at $R i_{h}=O\left(10^{-1}\right)$. Further experiments are required to assess whether this threshold is independent on $R e_{h}$.

- The critical Richardson $R i_{h}$ number for which buoyancy effects become noticeable is estimated. A first estimate taken from the literature for a vertical flat plate underpredicts the onset by one order of magnitude. A second estimate based on a dimensional analysis of a simplified version of the Navier-Stokes equations seems to match the experimental results in a better way, at least in an order of magnitude sense. 


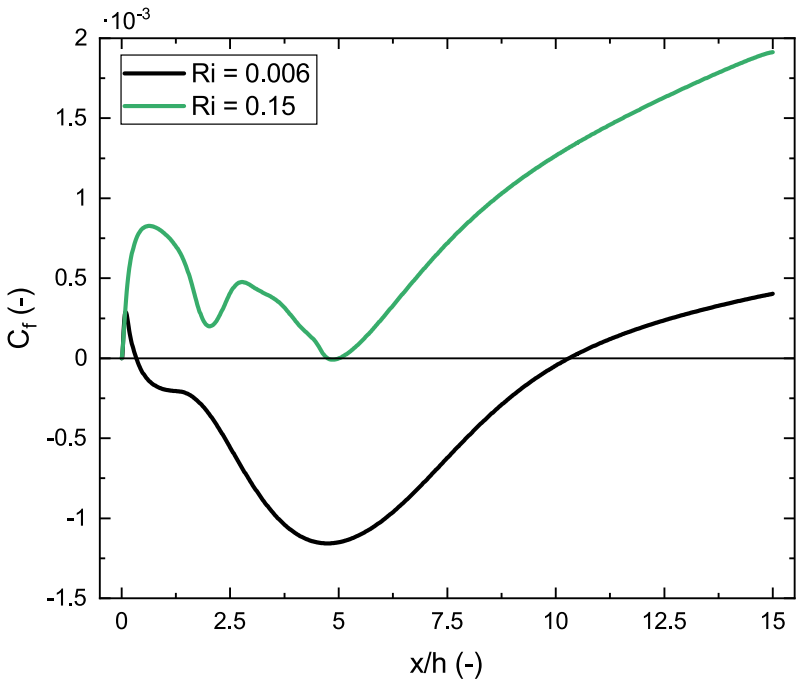

Fig. 15 Skin friction coefficient along the heating plate center-plane for the forced and mixed convection cases

- A local Nusselt number $N u$ asymmetry was observed the heating plate. Although the asymmetry is not far from experimental uncertainty, an influence of the sensing thermocouples on the heating plate temperature distribution cannot be excluded.

- The heating plate local $N u$ shows a great sensitivity on the assumed wall thermal boundary condition. This also has an impact on the physical interpretation to be given to $N u$ and the capability of $N u$ as an indicator to measure the onset to mixed convection.

- Different as for BFS cases with a constant specific heat flow physics, as strong three-dimensional effects due to the influence of the side walls are expected.

\section{Appendix 1: uncertainty analysis}

\section{Mean velocity measurements}

The uncertainty analysis is made following the recommendations of the Guide to the Expression of Uncertainty in Measurement (GUM) of the Joint Committee for Guides in Metrology (JCGM).

The measured signal velocity is given by

$V_{S S}=\mathrm{Ku}_{\mathrm{vol}}+S_{e f f} \Delta T+V_{0}$

where $V_{S S}$ is the measured voltage over the thermocouple sheaths, $\mathrm{K}$ is the calibration constant of the probe to be determined experimentally, $\mathrm{u}_{\mathrm{vol}}$ is the liquid metal volumetricaveraged flow velocity near the probe, $S_{\text {eff }}=\left(S_{S S}-S_{\text {GaInSn }}\right)$ can be interpreted as an effective Seebeck-coefficient for the probe, where $S_{S S}$ and $S_{G a I n S n}$ are the Seebeck coefficients of stainless steel (SS) and GaInSn, and $\Delta T=T_{2}-T_{1}$ where $T_{1}$ are the temperatures measured by thermocouples $T C 2$ and $T C 1$ and $V_{0}$ is the offset of the measurement.

Rearranging and averaging in time, one obtains the measurement equation for the mean velocity,

$f=\bar{u}=\frac{\bar{V}_{S S}-\bar{V}_{0}-S_{\text {eff }} \Delta T}{K}$

The combined uncertainty $u_{\bar{u}}$ for $\bar{u}$ is then

$u_{\bar{u}}=\sqrt{\left(\frac{\partial f}{\partial \bar{V}_{S S}} s_{\bar{V}_{S S}}\right)^{2}+\left(\frac{\partial f}{\partial \bar{V}_{0}} s_{\bar{V}_{0}}\right)^{2}+\left(\frac{\partial f}{\partial S_{e f f}} s_{S_{e f f}}\right)^{2}+\left(\frac{\partial f}{\partial \Delta T} s_{\Delta T}\right)^{2}+\left(\frac{\partial f}{\partial \mathrm{K}} s_{\mathrm{K}}\right)^{2}+\left(s_{\mathrm{r}}\right)^{2}}$

flux boundary condition, there is no obvious relationship between $N u$ and the mean reattachment point $x_{R}$ of the flow.

- The main uncertainty contributors for the velocity measurements relate to wetting issues during the calibration of the probes and to the limited samples of the repeatability study. Hence, the extended uncertainty for these measurements was evaluated calculating the effective degrees of freedom and using the t-Student distribution for each data point.

Future experiments are planned for the local measurement of $u^{\prime 2}, T, T^{\prime 2}$ and $u^{\prime} T^{\prime}$, for which minor instrumentation connection changes and facility optimizations are needed. High fidelity numerical simulations of the experiment are further needed to draw a more comprehensive picture of the where $\frac{\partial f}{\partial x_{i}}$ are the sensitivities of each uncertainty contributor, $s_{i}$ the standard uncertainties of each uncertainty contributor, where $s_{R}$ represents the standard uncertainty of the repeatability study.

\section{Determination of $s_{\bar{v}_{s s}}$}

$s_{\bar{V}_{S S}}$ is the statistical uncertainty due to the limited sampling time of $\bar{V}_{S S}$. Nobach and Tropea (2007) describe the methodology on how to calculate this uncertainty. Since $\bar{V}_{S S}$ is the measured signal proportional to the volume-averaged velocity around the probe tip, the integral time scale $t_{\text {int }}$ and the turbulence intensity $T u$ of the flow at each point should be measured. The calculation of $T u$ from the measured signal is straightforward. However, the measurement of $t_{i n t}$ on each measured point is not practically possible. In the absence of 
other practical alternative, $t_{\text {int }}$ was calculated from an estimation for the integral length scale $l_{\text {int }}$ assuming the Taylor hypothesis of frozen turbulence taking the local measured velocity as the characteristic velocity.

For probes $P 1$ and $P 2$, an approximate value for the integral length scale is calculated. Bailly and Comte-Bellot (2015) indicate that for a fully developed turbulent channel flow between two plates the integral length scale $l_{\text {int }}$ can be estimated as 0.6 times the half channel width. For the rest of the probes, $l_{\text {int }}$ was estimated taking the step height.

The statistical uncertainty $\epsilon$ of each point is then calculated as

$\epsilon=\sqrt{\frac{2 t_{i n t}}{t_{s}}} \cdot T u$

where the sampling time $t_{s}$ for each point was $t_{s}=105(s)$.

This uncertainty contributor is included into Eq. 15 as a type $A$ evaluation.

\section{Determination of $s_{\bar{v}_{0}}$}

Before every measurement campaign, the LTT24 system (and all other instrumentation) are warmed up for at least 12(h). Before every measurement, the LTT24 system is offset corrected. Nevertheless, the zero level of each probe is not totally stable over time, particularly considering that each measurement campaign (one parameter set) lasted approximately $10(h)$. A representative worst-case-scenario value for the fluctuation of all probes during the whole measurement campaign is estimated as $s_{\bar{V}_{0}}=60(n V)$. This uncertainty contributor is included into Eq. 15 as a type B evaluation assuming a rectangular distribution.

\section{Determination of $s_{s_{\text {eff }}}$}

The effective Seebeck coefficient is determined from the Seebeck coefficients of stainless steel (thermocouple sheaths) and GaInSn. In the absence of any other alternative, $S_{S S}$ is assumed as exact. Its value is obtained from Bentley (1998). The value for $S_{G a I n S n}$ and $s_{S_{\text {Galns }}}$ is taken from Plevachuk et al. (2014). The value for $s_{S_{e f f}}$ is estimated as $s_{S_{\text {eff }}}=s_{S_{\text {GalnSn }}}$.

\section{Determination of $s_{\Delta T}$}

$s_{\Delta T}$ is determined in the same manner as $s_{\bar{V}_{S S}}$. The big question to be answered is what the correct integral time scale for this quantity is. The key is to recognize that it is not each temperature $T_{1}$ and $T_{2}$ that is important here, but their difference. And a temperature difference between these two points is given only by flow structures smaller than the probe tip.
Hence, the integral length scale the worst-case value of $l_{\text {int }}=d_{\text {tip }}$ is taken.

This uncertainty contributor was included into Eq. 15 as a type $A$ evaluation.

\section{Determination of $S_{K}$}

The calculation of $s_{\mathrm{K}}$ is treated in detail in Schaub et al. (2021). Here, only the final values are shown:

\begin{tabular}{lll}
\hline & $K(\mu V / \mathrm{m} / s)$ & $s_{K}(\mu V / \mathrm{m} / \mathrm{s})$ \\
\hline P1 & 52.41 & 0.85 \\
\hline P2 & 50.85 & 0.57 \\
P3 & 41.95 & 3.21 \\
P4 & 23.18 & 4.25 \\
P5 & 43.97 & 1.45 \\
P6 & 54.82 & 1.99 \\
\hline
\end{tabular}

\section{Determination of $S R$}

As mentioned before, the measurement of one parameter set, i.e. the measurement of all velocity profiles plus the Nusselt number distribution along the heating plate, took about $10(h)$, i.e., a full working day. According to the GUM, a repeatability study should have at least 30 samples. This would take a minimum of 6 weeks to accomplish. Hence, only 3 samples were measured.

This uncertainty contributor was included into Eq. 15 as a type $A$ evaluation.

\section{Calculation of the extended uncertainty for $u_{\bar{u}}$}

From Eq. 15, one can calculate the extended uncertainty $u_{e, \bar{u}}$ for $u_{\bar{u}}$. Usually, one would take the coverage factor $k=2$, i.e., $u_{e, \bar{u}}=2 u_{\bar{u}}$ for a $95.45 \%$ confidence interval. However, due to the limited samples for $s_{\mathrm{R}}$ this cannot be made. Instead, the t-Student distribution must be used to obtain the coverage factor $k$. The effective number of degrees of freedom is calculated for every point with the Welch-Satterthwaite formula. For almost every measurement point, the calculated coverage factor is higher than 2 .

\section{Main uncertainty contributors}

The trend for the influence of each uncertainty contributor is the same for all measurement points. The main uncertainty contributors correspond to $s_{\bar{V}_{S S}}, s_{\bar{V}_{0}}, s_{\mathrm{K}}$ and $s_{\mathrm{r}}$. Depending on the local conditions, the influence of each contributor varied. The uncertainty contributors for $\bar{V}_{S S}$ and the repeatability can be improved by just taking longer measurements per 
point and by performing an exhaustive repeatability study. It must be noted that if longer measurements per point are desired, the spatial resolution of the profiles must be decreased to keep the daily measurement campaign extension within the laboratory opening hours. The contributor for $\bar{V}_{0}$ cannot be avoided. This is intrinsic to the measurement system and the authors are not aware of any commercially available measurement system with better performance than the LTT24. The contributor for $K$ can be improved by calibrating the probes in situ. This is, however, limited by the geometry of the test section.

\section{Nusselt number measurements}

The measurement equation reads

$f=N u=\frac{\alpha l_{c}}{\lambda_{\text {ref }}}=\frac{\dot{q} l_{c}}{\lambda_{\text {ref }} \Delta T}$

where $\Delta T=T_{w}-T_{b}$.

The standard uncertainty $u_{N u}$ for $N u$ is

$u_{N u}=\sqrt{\left(\frac{\partial f}{\partial \dot{q}} s_{\dot{q}}\right)^{2}+\left(\frac{\partial f}{\partial l_{c}} s_{l_{c}}\right)^{2}+\left(\frac{\partial f}{\partial \lambda_{\text {ref }}} s_{\lambda_{\text {ref }}}\right)^{2}+\left(\frac{\partial f}{\partial \Delta T} s_{\Delta T}\right)^{2}}$

where $\frac{\partial f}{\partial x_{i}}$ are the sensitivities of each uncertainty contributor and $s_{i}$ the standard uncertainties of each uncertainty contributor.

In this case, no term for the repeatability was included. For the heating plate, this uncertainty contributor can be neglected, since the average repeatability for all thermocouples mounted into the heating plate is in the order of $0.026^{\circ} \mathrm{C}$ for a $95.45 \%$ confidence interval.

\section{Determination of $s_{\dot{q}}$}

The values for $\dot{q}$ are calculated as $\dot{q}=\dot{Q} / A$, where $\dot{Q}$ is the power input and $A$ is the heating plate surface area. $\dot{Q}$ is calculated reading the power source display values for the DC voltage $V_{D C}$ and the DC current $I_{D C}$ as $\dot{Q}=V_{D C} \cdot I_{D C}$. After the measurement campaign, the display values were offset corrected by comparing the display values to actual measured values with a calibrated multimeter. As mentioned in Sect. 2.3.5, the energy balance for this experiment could not be precisely determined. This remains an open issue to be solved in future experiments.

The standard uncertainty $s_{\dot{q}}$ is taken from the power source data sheet and included into Eq. 18 as a type $B$ evaluation.

\section{Determination of $s_{l_{c}}$}

The uncertainty of the characteristic length, i.e., the step height, is the manufacturing tolerance of $0.1(\mathrm{~mm})$ and included into Eq. 18 as a type $B$ evaluation.

\section{Determination of $s_{\lambda_{\text {ref }}}$}

The value for $\lambda_{\text {ref }}$ as well that of $s_{\lambda_{\text {ref }}}$ were taken from Plevachuk et al. (2014) and are included as a type $B$ evaluation.

\section{Determination of $S_{\Delta T}$}

This contributor was determined in the same way as $s_{\bar{V}_{S S}}$ in Sect. 10.1.1. The integral time scale was calculated from an estimated integral length scale equal to the heating plate length, i.e., $l_{\text {int }}=15 \mathrm{~h}$. This contributor was included into Eq. 18 as a type $A$ evaluation.

\section{Calculation of the extended uncertainty for $\mathrm{u}_{\mathrm{Nu}}$}

In this case, since enough samples could be measured for each contributor obtained from a type $A$ evaluation, a coverage factor $k=2$ was used for a $95.45 \%$ confidence interval.

\section{Appendix 2: Setup details of the numerical calculations}

The steady-state Reynolds-averaged Navier-Stokes equations were solved in ANSYS CFX for the present test section (Fig. 2). The buoyancy term was modeled with the Boussinesq approximation. The conjugate heat transfer problem was numerically solved, i.e., by including the heating plate as a solid body in the computational domain. A constant specific heat flux $\dot{q}_{\text {const }}=\dot{Q} / A_{H P}$ was applied on the exterior surface of the heating plate, as in the experiment. The inlet boundary condition is a constant mass flow rate for $R e=10218$ with a constant velocity profile and inlet turbulence intensity of $1 \%$. Numerical schemes of first order were used for the momentum and energy equations (otherwise the solution would not converge), while the turbulence related quantities are calculated with second order schemes. The simulation convergence was assessed by letting the calculation run until all numerical residuals were below $10^{-6}$ and by judging local monitoring points of all relevant variables. In a geometry independency study, an outlet section extension of $30 h$ proved to be long enough. The numerical mesh has a value of $y^{+} \approx 1$ but at some local areas around the trip wire. A qualitative mesh independency study was performed 
and a mesh independent solution was achieved based on the local inspection of relevant variable profiles (mean velocity and temperature, turbulent kinetic energy, Reynolds stresses, etc.). The profiles were extracted at the same position as the permanent magnet probes are mounted in the test section. The Reynolds stress tensor was calculated with a baseline omega-based Reynolds stress model. The turbulent heat flux was modeled by calculating the turbulent Prandtl number $P r_{t}$ by means of the Kays correlation $P r_{t}=0.85+0.7 / P e_{t}$, where $P e_{t}=v_{t} / v$ is the turbulent Péclet number with $v_{t}$ the eddy kinematic viscosity and $v$ the liquid metal kinematic viscosity.

Author Contribution T. Schaub, W. Hering and R. Stieglitz contributed to the experiment conception. T. Schaub and F. Arbeiter contributed to the facility and/or the test section and/or the instrumentation conception and/or design and/or commissioning. T. Schaub planned and prepared the experimental campaign and the numerical calculations. T. Schaub ran the experiments and executed the numerical calculations. T. Schaub post-processed the data. T. Schaub and F. Arbeiter interpreted the data. T. Schaub wrote the first draft of the manuscript. All authors corrected previous versions of the manuscript. All authors read and approved the final manuscript.

Funding Open Access funding enabled and organized by Projekt DEAL. Financial support was provided by the EU-Horizon 2020 SESAME (Grant agreement ID: 654935) and ESFR-Smart (Grant agreement ID: 754501) projects.

Data availability The datasets generated during and/or analyzed during the current study are available from the corresponding author on reasonable request.

\section{Declarations}

Conflict of interest The authors have no conflicts of interest to declare that are relevant to the content of this article.

Open Access This article is licensed under a Creative Commons Attribution 4.0 International License, which permits use, sharing, adaptation, distribution and reproduction in any medium or format, as long as you give appropriate credit to the original author(s) and the source, provide a link to the Creative Commons licence, and indicate if changes were made. The images or other third party material in this article are included in the article's Creative Commons licence, unless indicated otherwise in a credit line to the material. If material is not included in the article's Creative Commons licence and your intended use is not permitted by statutory regulation or exceeds the permitted use, you will need to obtain permission directly from the copyright holder. To view a copy of this licence, visit http://creativecommons.org/licenses/by/4.0/.

\section{References}

Bailly C, Comte-Bellot G (2015) Turbulence. Springer International Publishing, Berlin. https://doi.org/10.1007/978-3-319-16160-0

Bentley RE (1998). Theory and practice of thermoelectric thermometry. In Handbook of Temperature Measurement. 3
Buckingham S (2018). Prandtl number effects in abruptly separated flows: LES and experiments on an unconfined backward facing step flow. Dissertation, UC Louvain. https://doi.org/10.35294/ phdt201811

Chen L, Asai K, Nonomura T, Guannan X, Lium T (2018) A review of backward-facing step (BFS) flow mechanisms, heat transfer and control. Thermal Sci Eng Progress 6:194-216. https://doi.org/10. 1016/j.tsep.2018.04.004

Da Vià R, Manservisi S (2019) Numerical simulation of forced and mixed convection turbulent liquid sodium flow over a vertical backward facing step with a four parameter turbulence model. Int J Heat Mass Transf 135:591-603. https://doi.org/10.1016/j.ijhea tmasstransfer.2019.01.129

De Brederode V, Bradshaw P (1972). Three-dimensional flow in nominally two-dimensional separation bubbles I. Flow behind a rearward facing step. I.C. Aero Report 71-19. Imperial College of Science and Technology

De Santis A, Shams A (2018) Application of an algebraic turbulent heat flux model to a backward facing step flow at low Prandtl number. Ann Nucl Energy 117:32-44. https://doi.org/10.1016/j. anucene.2018.03.016

Idelchik IE (2008) Handbook of Hydraulic Resistance, 4th edn. Revised and Augmented. Begell House Publishers Inc., U.S

Jäger W, Schumm T, Niemann M, Hering W, Stieglitz R, Magagnato F, Frohnapfel B, Fröhlich J (2017) Thermo-hydraulic flow in a sudden expansion. IOP Conf Series: Mater Sci Eng 228:012001. https://doi.org/10.1088/1757-899X/228/1/012001

Kapulla R (2000). Experimentelle Untersuchung von thermisch stratifizierten und unstratifizierten Mischungsschichten in Natrium und Wasser. Dissertation, ETH Zürich

Knebel JU, Krebs L, Müller U, Axcell BP (1998) Experimental investigation of a confined heated sodium jet in a co-flow. J Fluid Mech 368:51-79. https://doi.org/10.1017/S0022112098001463

Li X, Djilali N (1995) On the Scaling of Separation Bubbles. JSME Int j, Ser B 38(4):541-548. https://doi.org/10.1299/jsmeb.38.541

Lloyd JR, Sparrow EM (1970) Combined forced and free convection flow on vertical surfaces. Int J Heat Mass Transf 13(2):434-438. https://doi.org/10.1016/0017-9310(70)90119-5

Moffat RJ (1998) What's new in convective heat transfer? Int J Heat Mass Transf 19(2):90-101. https://doi.org/10.1016/S0142727X(97)10014-5

Nadge PM, Govardhan RN (2014) High Reynolds number flow over a backward-facing step: structure of the mean separation bubble. Exp Fluids 55:1657. https://doi.org/10.1007/s00348-013-1657-5

Niemann M, Fröhlich J (2016b). In: 11th international ERCOFTAC symposium on engineering turbulence modelling and measurements.

Niemann M, Fröhlich J (2016a) Buoyancy-affected backward-facing step flow with heat transfer at low Prandtl number. Int J Heat Mass Transf 101:1237-1250. https://doi.org/10.1016/j.ijheatmasstrans fer.2016.05.137

Nobach H, Tropea C (2007). Fundamentals of data processing. In Tropea C, Yarin A, Foss J (eds.) Springer Handbook of Experimental Fluid Dynamics, Springer-Verlag Berlin Heidelberg, pp 1399-1417 https://doi.org/10.1007/978-3-540-30299-5

Oder J, Shams A, Cizelj L, Tiselj I (2019) Direct numerical simulation of low-Prandtl fluid flow over a confined backward facing step. Int J Heat Mass Transf 142:118436. https://doi.org/10.1016/j.ijhea tmasstransfer.2019.118436

Pacio J, Daubner M, Fellmoser F, Wetzel T (2019) Experimental study of the influence of inter-wrapper flow on liquid-metal cooled fuel assemblies. Nucl Eng Des 352:110145. https://doi.org/10.1016/j. nucengdes.2019.06.007

Plevachuk Y, Sklyarchuk V, Eckert S, Gerbeth G, Novakovic R (2014) Thermophysical properties of the liquid Ga-In-Sn eutectic alloy. 
J Chem Eng Data 59(3):757-763. https://doi.org/10.1021/je400 $882 \mathrm{q}$

Schaub T, Wüstling S, Konrad J, Tasler M (2021) Design and calibration of permanent magnet probes for the local measurement of velocity and temperature in a liquid metal backward facing step flow. Exp Fluids 62:210. https://doi.org/10.1007/ s00348-021-03293-6

Schumm T, Frohnapfel B, Marocco L (2018) Investigation of a turbulent convective buoyant flow of sodium over a backward- facing step. Heat Mass Transf 54:2533-2543. https://doi.org/10.1007/ s00231-017-2102-8

Sobolev V (2011). Database of thermophysical properties of liquid metal coolants for GEN-IV. BLG Open Report Series of the Belgian Nuclear Research Centre, Dec 2011. Report number BLG-1069

Star S, Stabile G, Rozza G, Degroote J (2021) A POD-Galerkin reduced order model of a turbulent convective buoyant flow of sodium over a backward-facing step. Appl Math Model 89(1):486-503. https:// doi.org/10.1016/j.apm.2020.07.029

Stephan P, Kabelac S, Kind M, Mewes D, Schaber K, Wetzel T (eds) (2019) VDI Wärmeatlas. https://doi.org/10.1007/ 978-3-662-52989-8

Straub S, Forooghi P, Marocco L, Wetzel T, Vinuesa R, Schlatter P, Frohnapfel B (2019) The influence of thermal boundary conditions on turbulent forced convection pipe flow at two Prandtl numbers. Int J Heat Mass Transf 144:118601
Vinuesa R, Schlatter P, Nagib M (2018) Secondary flow in turbulent ducts with increasing aspect ratio. Phys Rev Fluids 3:054606. https://doi.org/10.1103/PhysRevFluids.3.054606

von Weissenfluh T, Sigg B (1988). Experience with permanent magnet probes for the measurement of local velocities in liquid metals. In Unger Y, Mond M, Branover H (eds.) Liquid Metal Flows: Magnetohydrodynamics and Application. https://doi.org/10.2514/4. 865862

von Weissenfluh T (1985) Probes for local velocity and temperature measurements in liquid metal flow. Int J Heat Mass Transf 28(8):1563-1574. https://doi.org/10.1016/0017-9310(85)90258-3

Wang C, Zhao P, Lei M, Lu K, Ge Z, Liu J, Li Y, Pei G (2020) DNS of instantaneous behavior in turbulent forced and mixed convection of liquid metal past a backward-facing step. Flow Turbul Combust 107:125-147. https://doi.org/10.1007/s10494-020-00214-1

Weigand B (2015). Analytical methods for heat transfer and fluid flow problems. 2nd edition. Springer, Berlin, Heidelberg. https://doi. org/10.1007/978-3-662-46593-6

Woodfield PL, Monde M, Mitsutake Y (2006) Improved analytical solution for inverse heat conduction problems on thermally thick and semi-infinite solids. Int J Heat Mass Transf 149(17-18):2864 2876. https://doi.org/10.1016/j.ijheatmasstransfer.2006.01.050

Publisher's Note Springer Nature remains neutral with regard to jurisdictional claims in published maps and institutional affiliations. 